\title{
OPEN Synaptic AP2 CCV life cycle regulation by the Eps15, ITSN1, Sgip1/AP2, synaptojanin1 interactome
}

\author{
R. Mishra ${ }^{1,3}$, G. F. Sengül1 ${ }^{1}$, E. Candiello ${ }^{1,2} \&$ P. Schu ${ }^{1 凶}$
}

The AP1/б1B knockout causes impaired synaptic vesicle recycling and enhanced protein sorting into endosomes, leading to severe intellectual disability. These disturbances in synaptic protein sorting induce as a secondary phenotype the upregulation of AP2 CCV mediated endocytosis. Synapses contain canonical AP2 CCV and AP2 CCV with a more stable coat and thus extended life time. In AP1/б1B knockout synapses, pool sizes of both CCV classes are doubled. Additionally, stable CCV of the knockout are more stabilised than stable wt CCV. One mechanism responsible for enhanced CCV stabilisation is the reduction of synaptojanin 1 CCV levels, the PI-4,5- $\mathrm{P}_{2}$ phosphatase essential for AP2 membrane dissociation. To identify mechanisms regulating synaptojanin1 recruitment, we compared synaptojanin 1 CCV protein interactome levels and CCV protein interactions between both CCV classes from wt and knockout mice. We show that ITSN1 determines synaptojanin1 CCV levels. Sgip1/AP2 excess hinders synaptojanin1 binding to ITSN1, further lowering its levels. ITSN1 levels are determined by Eps15, not Eps15L1. In addition, the data reveal that reduced amounts of pacsin1 can be counter balanced by its enhanced activation. These data exemplify the complexity of CCV life cycle regulation and indicate how cargo proteins determine the life cycle of their CCV.

Synapses contain two classes of AP2 CCV (adaptor-protein complex 2; clathrin-coated-vesicles). Canonical AP2 CCV, canCCV, and AP2 CCV with a stabilised protein coat and thus a longer half live. Stable CCV account for about $15 \%$ of synaptic AP2 $\mathrm{CCV}^{1,2}$. In synapses of the AP1/ $1 \mathrm{~B}$-adaptin knockout mouse (ko) numbers of both classes increase twofold. In addition, the stable AP2 CCV in ko mouse synapses are even more stabilised $\mathrm{CCV}$, hereafter stCCV ${ }^{1}$, compared to wt stable CCV, as summarised in Fig. 1. AP2 stCCV do not endocytose synaptic vesicle (SV) proteins. They are enriched in the synaptic active zone (AZ) proteins stonin2 and Git $1^{1}$. These are organised in circles around AZ. Stonin2, an AP2 co-adaptor CCV protein, recruits Git ${ }^{3-6}$. Deficiency in presynaptic Git1 leads to impaired SV recycling and transsynaptic signaling ${ }^{3}$. The molecular mechanisms are not known. Git1 transport via stCCV points to important functions of stCCV in the regulation of AZ plasticity ${ }^{1}$.

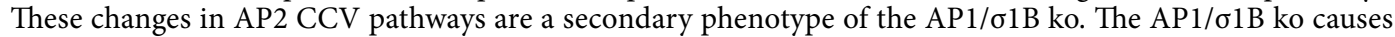
slowed down and incomplete SV recycling and enhanced endolysosomal protein sorting ${ }^{1,2,7,8}$. Mice and humans deficient in the X-chromosome encoded AP1 subunit $\sigma 1 \mathrm{~B}$ have severe mental retardation disease and deficits in motor $\mathrm{control}^{8}$. The alterations in the AP2 CCV pathways are expected to suppress the effects of disturbed synaptic protein sorting caused by AP1/ $11 \mathrm{~B}$-deficiency.

The AP1/ $1 \mathrm{~B}$ ko induced secondary phenotype in AP2 CCV pathways enables us to investigate physiological mechanisms regulating the AP2 CCV life cycle. CCV coat protein ko and protein domain overexpression studies revealed coat protein interactions and functions in CCV formation. However, CCV proteins interact with several proteins during the CCV life cycle via the same domains and thus these approaches analyse early steps in CCV formation, but they can not resemble physiological mechanisms regulating the CCV life cycle.

We identified three molecular mechanisms responsible for the enhanced stability of AP2 stCCV ${ }^{1}$. They affect all layers of a CCV: the outer clathrin basket, the inner AP2 layer and the membrane phospholipid composition. Compared to wt stable CCV, stCCV have less of the clathrin disassembly protein Hsc70, their AP2 complexes are hyperactivated for stable membrane binding and they have less of the PI-4,5- $\mathrm{P}_{2}$ phosphatase synaptojanin 1 .

${ }^{1}$ Department of Cellular Biochemistry, University Medical Center, Georg-August University Göttingen, Humboldtallee 23, 37073 Göttingen, Germany. ${ }^{2}$ Institute for Cancer Research and Treatment (IRCC), Turin, Italy. ${ }^{3}$ Present address: Department of Clinical Neurosciences, John Van Geest Centre for Brain Repair, University of Cambridge, Cambridge, England, UK. ${ }^{凶}$ email: pschu@gwdg.de 
A wt synapse
AP2 CCV
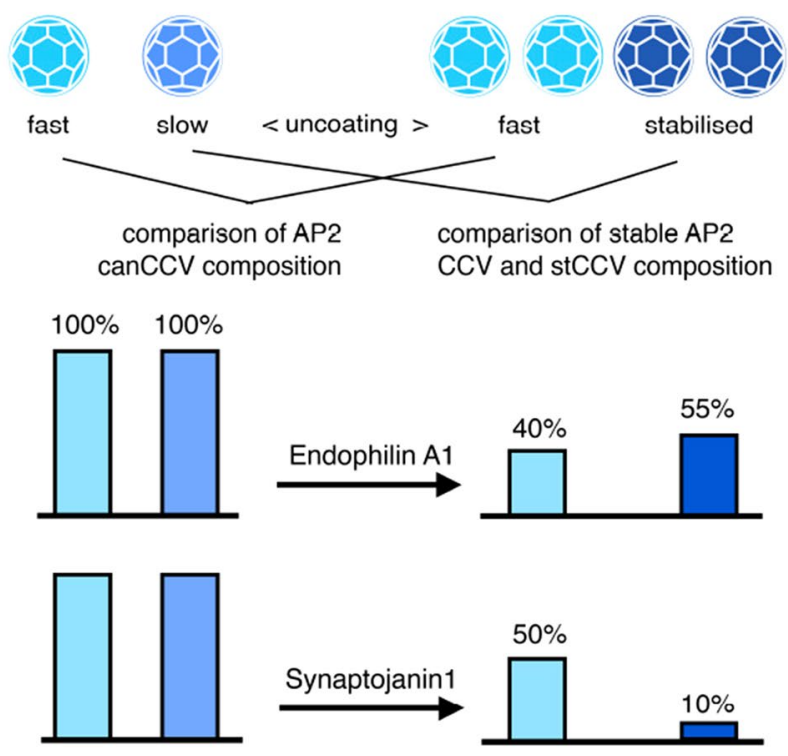

B

Eps15L1

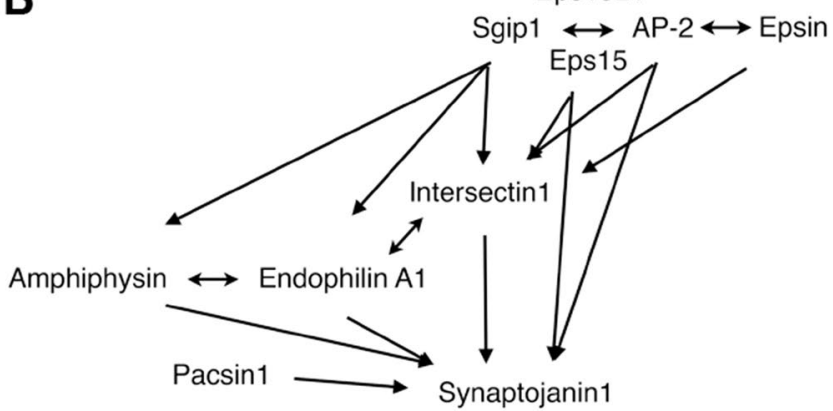

Figure 1. (A) Summary of alterations in the synaptic AP2 CCV pools, induced by impaired SV recycling due to AP1/ $1 \mathrm{~B}$-adaptin deficiency. Pool sizes of canonical canCCV and stable CCV are increased twofold and in addition, stable, slow uncoating CCV become more stabilised (stCCV) Bar diagrams indicate the protein reduction per CCV for endophilin and synaptojanin1. (B) The synaptojanin1 interactome of CCV proteins and their interactions. Clathrin and dynamin are not included. Proteins listed at the top appear first at a AP2 CCP. Both Eps15 proteins bind synaptojanin1. Pacsin1 belongs to the group of proteins recruited late during CCV budding.

AP2 hyperactivation is caused by an increase in AAK1 (adaptin-associated kinase 1), which phosphorylates $\mu 2$. This stabilises the AP2 open state, enabling $\mu 2$ to bind PI-4,5- $\mathrm{P}_{2}$ and also cargo ${ }^{9-12}$. A stCCV contains with $10 \%$ of wt only residual amounts of synaptojanin 1. PI-4,5- $\mathrm{P}_{2}$ dephosphorylation by synaptojanin 1 is essential for AP2 membrane dissociation ${ }^{13-19}$. Synaptojanin 1 catalytic activity is stimulated by high membrane curvature . $^{20}$. Therefore, its CCV uncoating activity is regulated by its recruitment into CCV. In this study, we present our analysis of the mechanisms regulating synaptojanin1 levels in AP2 CCV.

Understanding the mechanisms regulating synaptojanin 1 activities is not only important for CCV life cycle regulation, but is also essential to understand the development of several severe neurological diseases. Synaptojanin 1 deficiencies cause neonatal refractory epilepsy and neurodegeneration ${ }^{21}$. Point mutations have been identified in patients with early onset Parkinson's disease, intractable epilepsy and tau pathology ${ }^{22-26}$. Three forms of Alzheimer's disease are associated with elevated synaptojanin1 levels ${ }^{27-29}$. Loss of its 5- and 4-phosphatase activities leads to early onset refractory seizures and neurological decline ${ }^{21}$. Its overexpression is associated with Down syndrome ${ }^{30,31}$. Its SAC1 domain dephosphorylates PI-4-P, but is also able to dephosphorylate PI-3-P and PI-3,4- $\mathrm{P}_{2}$, both of which regulate endosomal dynamics and autophagosome formation. Synaptojanin1 deficiencies induce strong phenotypes in SV recycling, whereas autophagosomes are not abundant in mature synapses ${ }^{32,33}$.

The synaptojanin 1 CCV interactome consists of 9 proteins. Eight of these have interactions with each other, as summarised in Fig. 1. Synaptojanin 1 can be bound by proteins appearing early at the site of CCV formation 
and also by proteins appearing late, just before membrane scission. Early proteins are AP2, Eps15, Eps15L1 (Eps15-like 1, also known as Eps15R) and clathrin ${ }^{34-36}$. Proteins appearing later are endophilin A1, amphiphysin, intersectin 1 (ITSN1) and pacsin 1 (also known as syndapin1). They also bind each other and those early appearing proteins. These proteins are also bound by the early appearing proteins Sgip1 and epsin (Fig. 1) ${ }^{13,37-43}$. It is not understood, why synaptojanin 1 is bound by so many, ubiquitously expressed CCV proteins. This could ensure robust, continuous recruitment of synaptojanin 1. The late appearing endophilin A1 (hereafter just endophilin) recruits synaptojanin 1 just before membrane scission and thus this interaction has been considered to be the most relevant ${ }^{13,41,42}$. The late recruitment of an uncoating enzyme, whose catalytic activity is activated by high membrane curvature, also appears to be beneficial for CCV formation ${ }^{13,44-46}$.

Of all the synaptojanin 1 interacting CCV proteins, we already analysed amphiphysin and endophilin levels in wt and ko synaptic AP2 CCV in a previous study ${ }^{1}$. A stCCV contains $50 \%$ of endophilin compared to a stable CCV from wt mice, but only $10 \%$ of synaptojanin $1^{1}$. This indicates that endophilin does not determine the amount of synaptojanin 1 recruited into an AP2 CCV. However, phosphorylation of endophilin by LRRK2 kinase could reduce its binding of synaptojanin 1 causing the dramatic reduction in synaptojanin 1 stCCV levels ${ }^{40,47-51}$. Here we show that the levels and activities of the endophilin modifying kinase LRRK2 are not changed in stCCV compared to wt stable CCV, finally excluding endophilin as the synaptojanin 1 recruiter. To unravel the mechanisms regulating synaptojanin 1 CCV levels, we analysed ko canCCV and stCCV for alterations in the remaining synaptojanin 1 interactome members. ITSN1 CCV level determine synaptojanin1 CCV levels. Sgip1/ AP2 competition with synaptojanin1 for ITSN1 binding can lower synaptojanin 1 levels even further. ITSN1 CCV levels are determined by Eps15 CCV levels, but the highly homologous Eps15L1 is not involved. Thus, protein:protein ratios and competitive interactions determine the CCV life cycle. In addition, this study shows that a reduction in pacsin 1 can be compensated by its enhanced activation. This demonstrates the existence of complex pathways regulating the CCV life cycle. A model is proposed, which shows how co-adaptors and thus cargo proteins determine the life cycle of their AP2 CCV.

\section{Results}

Endophilin and LRRK2 do not determine synaptojanin1 CCV levels. We compare the protein content between the synaptic wt and ko canCCV as well as wt stable CCV and the ko stCCV. The numbers of both AP2 CCV classes are doubled in ko synapses ${ }^{1,2,8}$. Thus, a change in the amount of a protein in a single CCV is half of the change in the CCV pool (Fig. 1). Previously, we have shown that the synaptojanin 1 binder endophilin

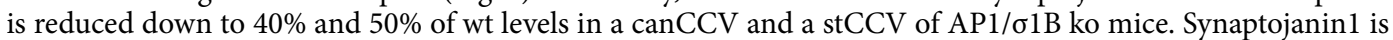
also reduced to $50 \%$ of wt in canCCV, but a stCCV has only $10 \%$ of wt synaptojanin 1 levels, despite $50 \%$ of wt endophilin levels ${ }^{1}$. This suggests that endophilin may not regulate the amount of synaptojanin 1 incorporated into CCV. However, data in the literature indicate, that endophilin-synaptojanin 1 binding might be differentially regulated by LRRK2 (leucine-rich repeat kinase) ${ }^{41,42,52-54}$. Endophilins BAR-domain binds the neck of a budding CCV, before membrane scission ${ }^{55}$, and its SH3-domain binds synaptojanin1 (Fig. 2A). The BAR-domain residue Ser75 is phosphorylated by LRRK2, but the function of this modification has not been tested directly ${ }^{53}$. It is expected, that Ser75 phosphorylation blocks membrane binding of the BAR-domain, but this would not necessarily inhibit endophilin recruitment to CCV, because its SH3-domain binds 6 CCV proteins: dynamin, intersectin1 (ITSN1), syndapin, VGLUT1, N-type $\mathrm{Ca}^{2+}$-channels and parkin ${ }^{56-60}$. If endophilin is recruited via its $\mathrm{SH} 3$-domain by CCV proteins, its BAR-domain can recruit the Arf6-GEF EFA6 ${ }^{61}$ (Fig. 2A). The order of these binding modes is not known. A competition of 7 proteins for endophilins SH3-domain could impair its ability to recruit synaptojanin1. LRRK2 activity could be increased in stCCV favouring its recruitment via protein-SH3 binding and leading to reduced synaptojanin 1 recruitment. Therefore, we compared LRRK2 levels and also its activation levels between the CCV from wt and ko mice.

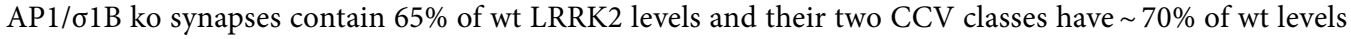
(Fig. 2), but LRRK2 activity could be stimulated in stCCV compared to wt stable CCV. LRRK2 has a GTPase domain and GTP binding activates kinase activity ${ }^{52}$. Importantly, the LRRK2 GEF ArhGEF7 binds Git1, which is enriched in stCCV, suggesting higher LRRK2 activity in stCCV ${ }^{1,3}$. The ArhGEF7 level is slightly increased

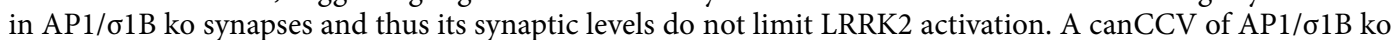
synapses has $60 \%$ of wt ArhGEF7 levels. A stCCV has $~ 42 \%$ of wt, indicating that stCCV LRRK2 is less active than canCCV LRRK2 (Fig. 2). The commercial anti-ArhGEF7 antibody labelled two protein bands in all fractions. The intensity of both changed in the same way. CCV were enriched in the fast migrating ArhGEF7 protein (Fig. 2C). LRRK2 phosphorylation, e.g. by PKA, on Ser935 leads to 14-3-3 protein binding and its inactivation (Fig. 2A) ${ }^{62,63}$. An AP1/ 1 1B ko synapse canCCV has $85 \%$ of wt LRRK2 Ser935-Pi-level. A stCCV has 55\% of wt LRRK2 Ser935Pi, but LRRK2 is also less activated by ArhGEF7. Thus, LRRK2 activation/inactivation cycles appear to be less active in stCCV compared to wt, stable CCV. The endophilin CCV levels and the LRRK2 data demonstrate that endophilin does not determine the amount of synaptojanin 1 in a CCV.

It is worth mentioning, that the changes in ko synapse LRRK2 levels are in line with their increase in endoso-

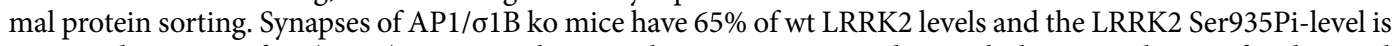
increased to $140 \%$ of wt (Fig. 2). Decreased LRRK2 kinase activity is in line with the upregulation of endosomal protein transport, because LRRK2 inhibits several Rab proteins which regulate endosomal protein transport ${ }^{52}$.

Pacsin1: reduced recruitment into stCCV, but enhanced activation. The synaptojanin1 binder pacsin1 (Fig. 1) has a F-BAR, a SH3 and an unstructured domain (Fig. 3A). Its SH3-domain interacts with dynamin and the actin polymerisation inducer N-WASP. Intramolecular interaction of F-BAR and SH3 domains causes pacsin 1 autoinhibition. The intermediate, unstructured sequence contains NPF-motifs, which mediate binding to EHD's (epsin-homology-domain) of CCV co-adaptor proteins ${ }^{64}$. Pacsin 1 autoinhibition is prevented 
A

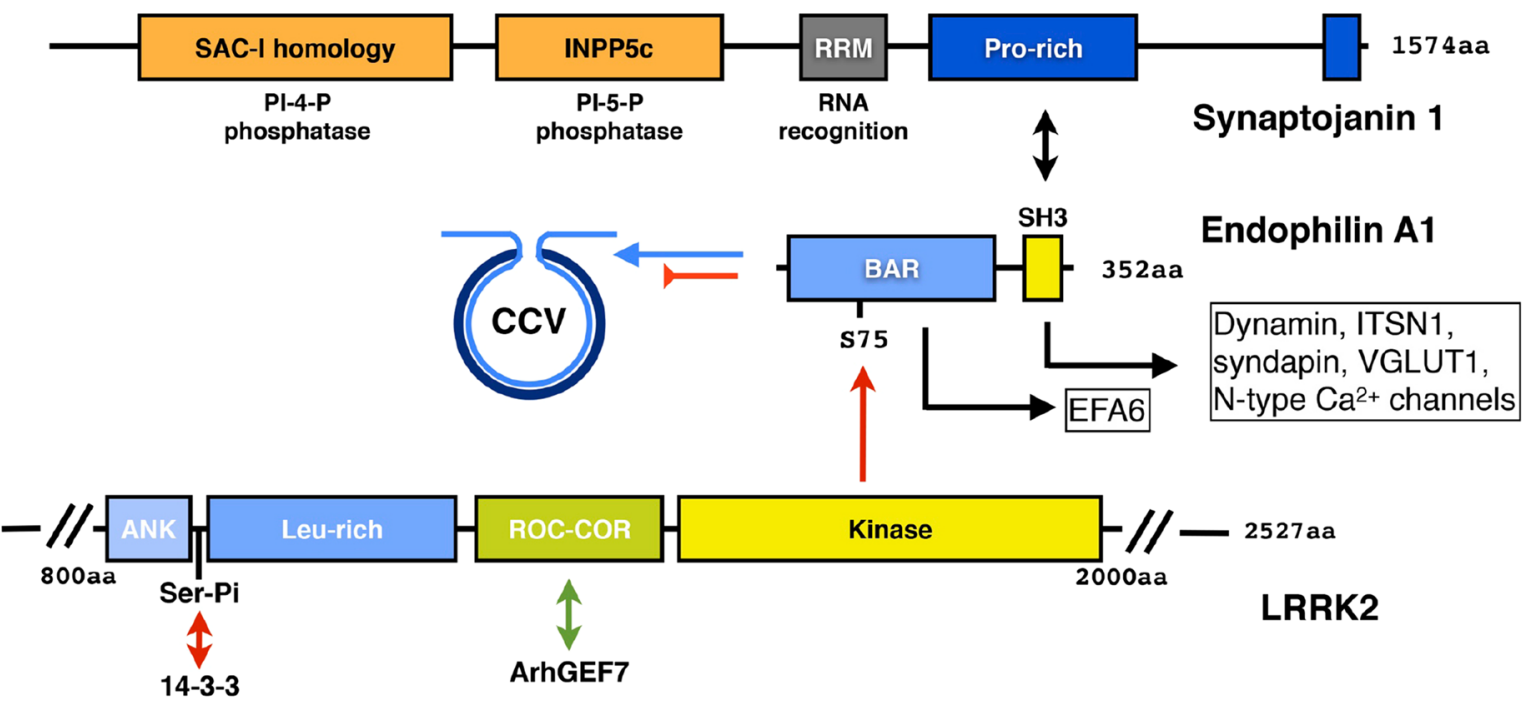

B

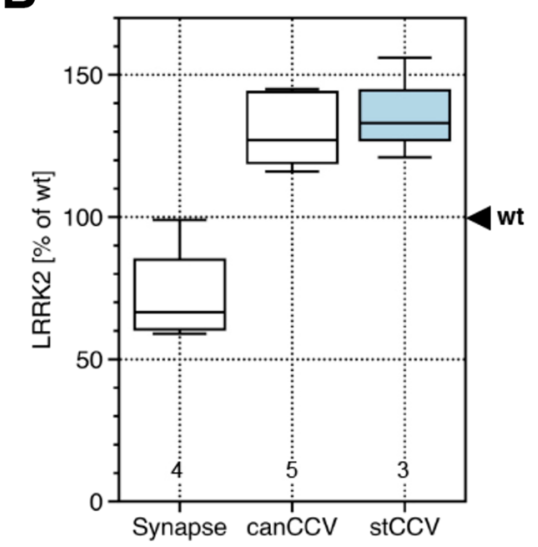

C

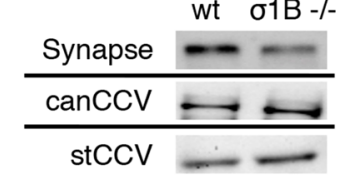

$\%$ of wt per CCV

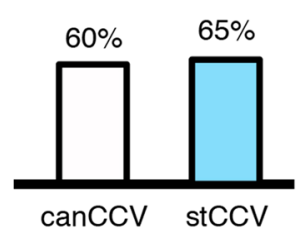

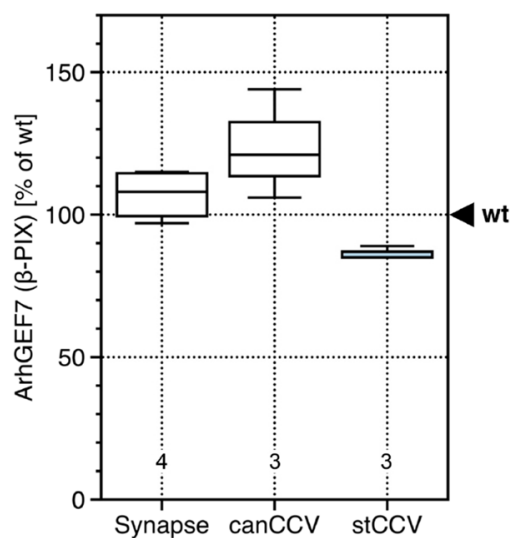

wt $01 \mathrm{~B}-1-$

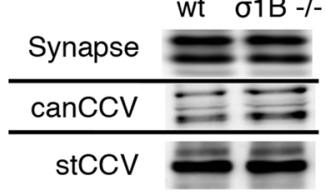

$\%$ of wt per CCV

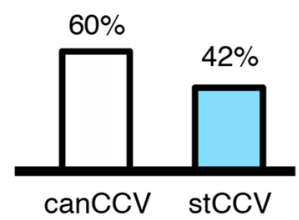

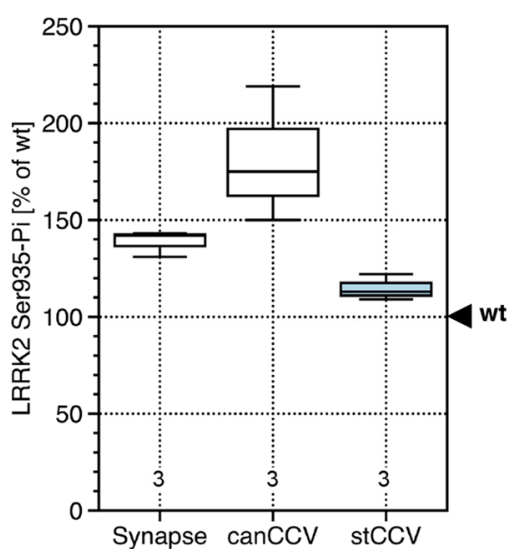

wt $\quad$ o1B -/-

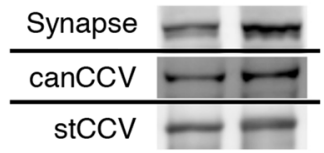

$\%$ of wt per CCV

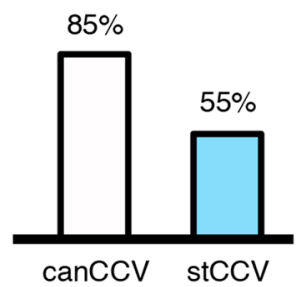

Figure 2. (A) CCV protein interactions of endophilin A1. Endophilin membrane binding to the neck of budding CCV, and synaptojanin1 recruitment, could be inhibited by LRRK2 (N-terminal armadillo and C-terminal WD40 domains are not depicted). If endophilin membrane binding is inhibited, it could also be recruited by binding of its $\mathrm{SH} 3$ domain to $\mathrm{CCV}$ proteins. Green arrow indicates activating, red arrows inhibitory reactions. (B) Comparison of LRRK2 protein ( $240 \mathrm{kDa}$ band) and activity (ArhGEF7, $74 \mathrm{kDa}$ band; LRRK2 Ser935-Pi, $240 \mathrm{kDa}$ band) levels between synapses and synaptic AP2 canCCV and wt, stable CCV and stCCV pools from wt and AP1/o1B-adaptin ko mice. Values from wt samples are defined as $100 \%$ and values from ko samples are expressed relative to wt. Numbers in box-blot diagrams indicate the number of independent biological samples. (C) Representative western-blot data used for the box-blot diagram (see "Materials and methods" section for details). (D) Bar scheme below indicates changes in protein levels per CCV. 

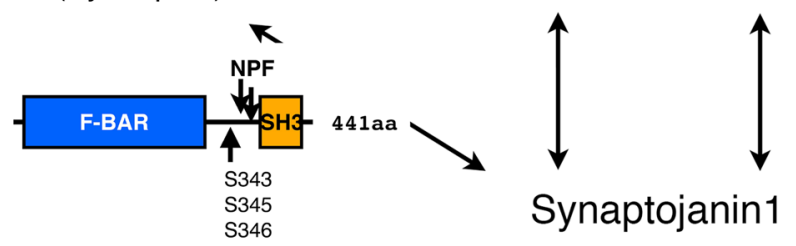

B
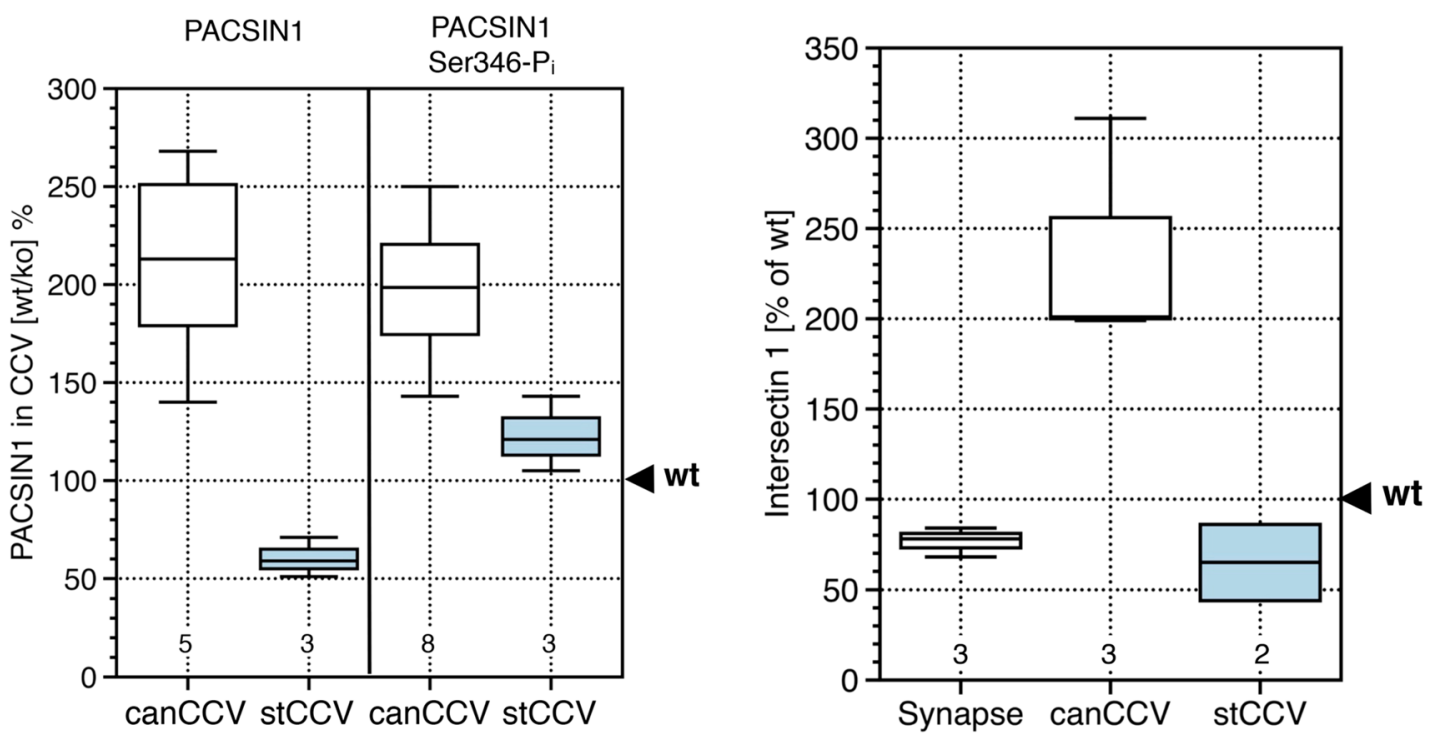

\section{C}

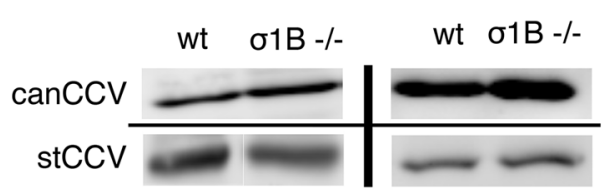

D

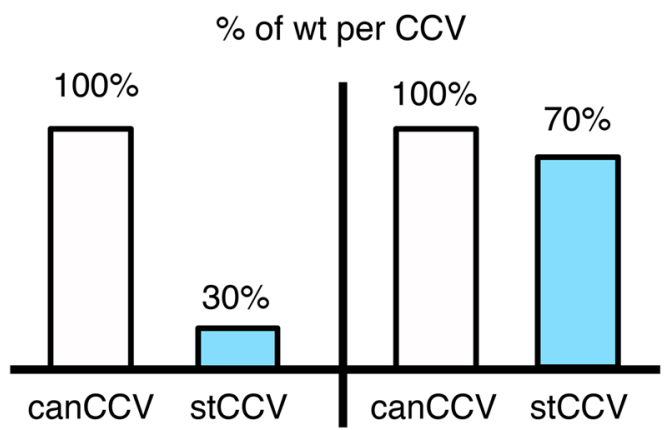

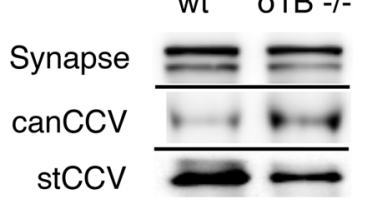

$\%$ of wt per CCV

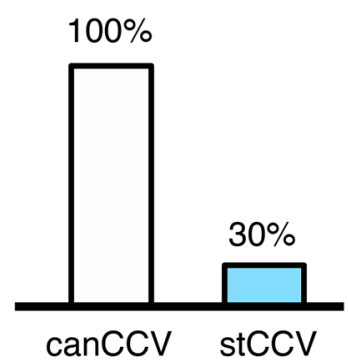

Figure 3. Pacsin1, activated pacsin1 (Ser346-Pi) and ITSN1 wt versus ko synaptic and CCV levels. (A) ProteinProtein interaction scheme of these synaptojanin1 binders. (B) Comparison of pacsin1, phosphorylated and

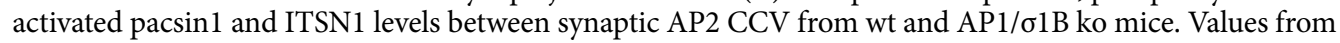
wt samples are defined as $100 \%$ and values from ko samples are expressed relative to wt. Numbers in box-blot diagrams indicate the number of independent biological samples. (C) Representative western-blot data used for the box-blot diagram (see "Materials and methods" section for details) Pacsin1, $52 \mathrm{kDa}$ band; Pacsin1 Ser346-Pi, $52 \mathrm{kDa}$ band; ITNS1, $200 \mathrm{kDa}$ band;. (D) Bar scheme below indicates changes in protein levels per CCV.

by serine phosphorylation in this unstructured sequence. Ser343 is phosphorylated by Pak5, which also phosphorylates the proline-rich domain of synaptojanin1, with which it binds endophilins SH3 domain. Either of the two phosphorylations stabilises pacsin1-synaptojanin1 binding ${ }^{39,50,65-70}$.

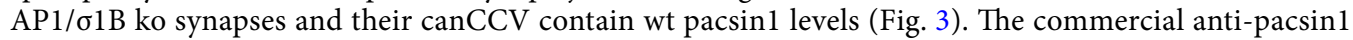
antibody detected besides the major protein band a second band of very low intensity. Its changes in intensities between wt and ko samples matched those of the major band (Fig. 3C). A stCCV contains only 30\% of wt, stable 
A

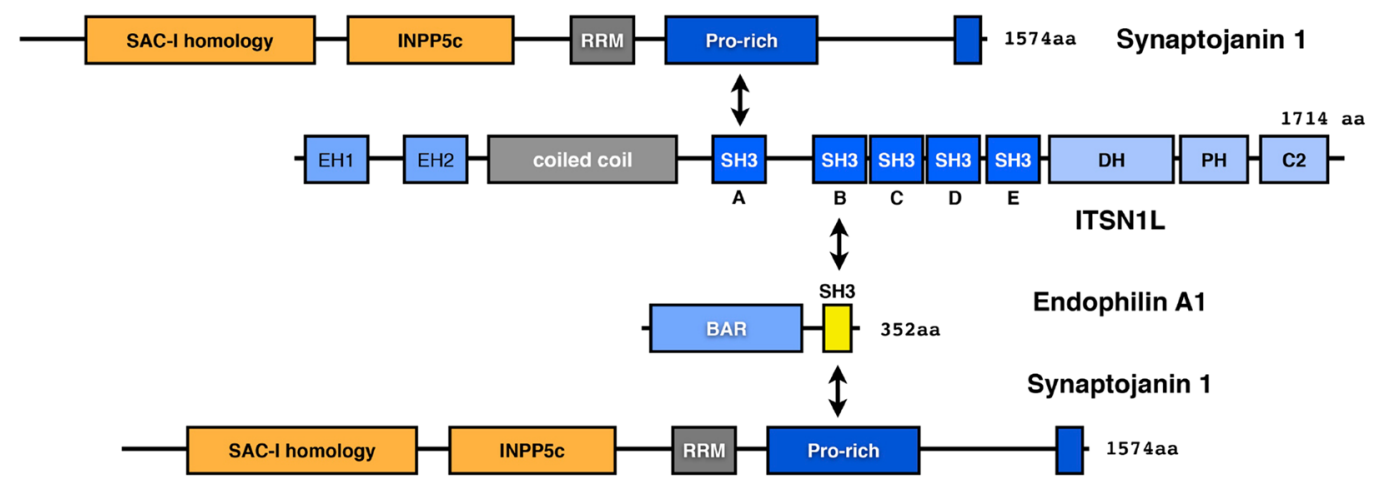

B
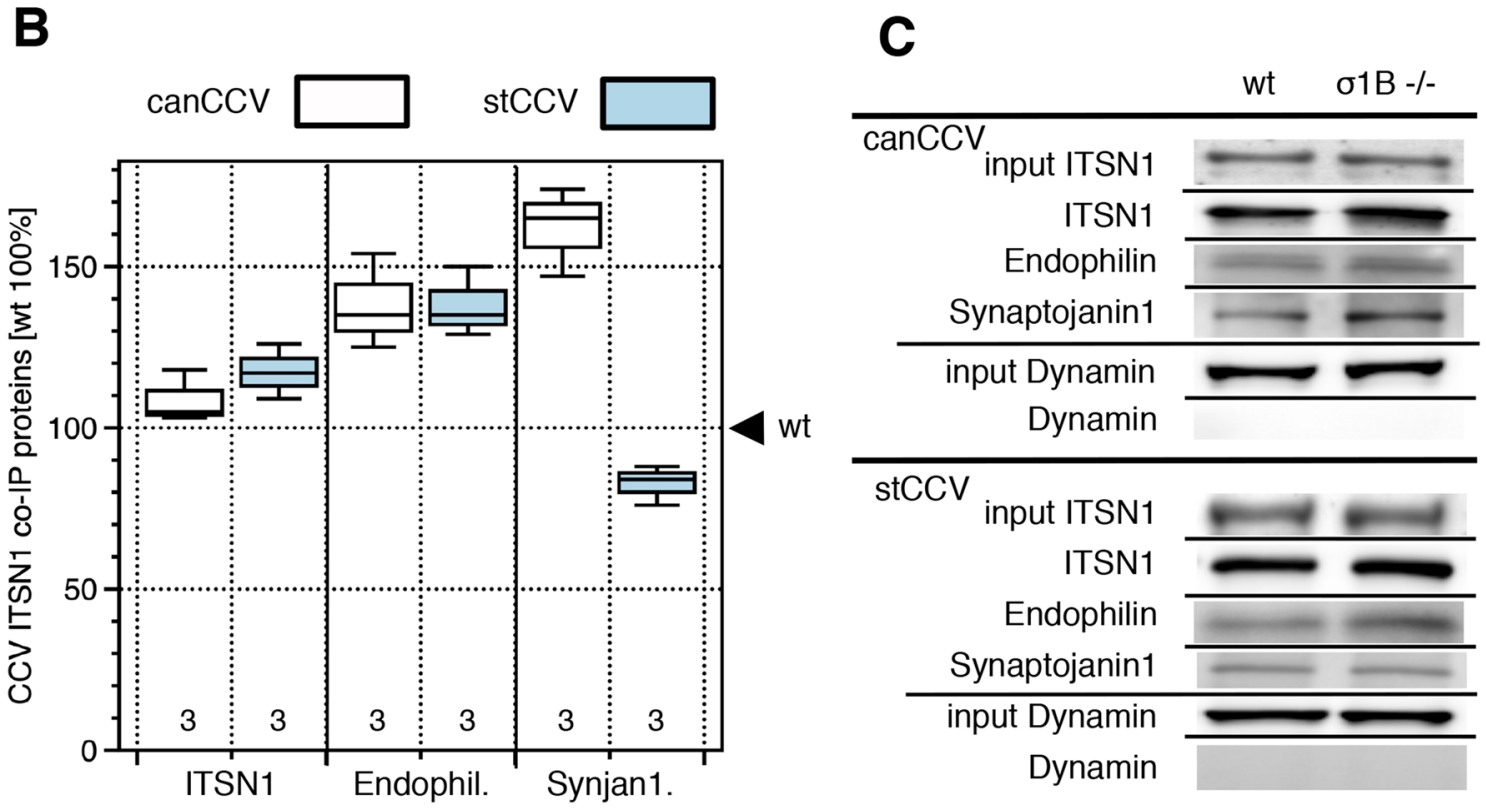

Figure 4. (A) Interaction of synaptojanin1 with ITSN1 and endophilin and of endophilin with ITSN1. (B) ITSN1 binding ratios of endophilin and synaptojanin1 of wt and ko canCCV and wt stable CCV and ko stCCV measured by ITSN1 immunoprecipitation after CCV coat protein solubilisation. Numbers indicate the number of independent experiments performed. (C) Representative western-blot experiments (see "Materials and methods" section for details). ITSN1, $200 \mathrm{kDa}$ band; Endophilin, $37 \mathrm{kDa}$ band; Synaptojanin1, $145 \mathrm{kDa}$ band; Dynamin, $95 \mathrm{kDa}$ band.

CCV pacsin 1 . This reduction could be responsible for the reduction in synaptojanin 1 levels, but stCCV pacsin 1 could also have a higher phosphorylation level.

Using a commercial pacsin1 Ser346-Pi antibody, we found wt pacsin1-Pi levels in ko mice canCCV (Fig. 3). A stCCV has $65 \%$ of wt pacsin $1-\mathrm{Pi}$, despite the pacsin 1 reduction down to $30 \%$ of wt (Fig. 3 ). Thus, the fraction of activated pacsin 1 in a stCCV is about twofold higher than in a wt, stable CCV. We can not exclude, that the reduction in the stCCV pacsin 1 level does not contribute at all to the reduced amount of synaptojanin 1 in stCCV. However, the compensation of reduced pacsin 1 stCCV levels by its increased activation is not in line with a pacsin 1 function in synaptojanin 1 recruitment. These data demonstrate a quality control mechanism for the CCV life cycle regulation. The kinase remains to be determined.

ITSN1 is differentially recruited to CCV classes. ITSN1 is able to bind synaptojanin1 and endophilin simultaneously and independent of each other. It has been proposed, that ITSN1 is a platform for coordinating synaptojanin1 recruitment by endophilin (Fig. 3A) ${ }^{58}$. ITSN1 appears early at budding CCV and its level increases continuously during budding. It could mediate an even synaptojanin 1 distribution, supporting fast PI4,5- $\mathrm{P}_{2}$ dephosphorylation. Brain ITSN1, ITSN1L, has a C-terminal extension of RhoGEF, PH and C2 domains. They are preceded by five SH3 domains (Fig. 4A). ITSN1L (hereafter just ITSN1) is constitutively active due to a five amino acid insertion in the SH3A domain ${ }^{71,72}$. ITSN1 SH3A binds synaptojanin1, SH3B binds endophilin. The linking sequence binds AP2 (Fig. 4A) ${ }^{73,74}$. AP1/ $\sigma 1 \mathrm{~B}$ ko mice synapses contain $80 \%$ of wt ITSN1 levels. The commercial ITSN1 antibody detects also a low intensity, faster migrating protein in synapses. It is also present at $80 \%$ of wt levels (Fig. 3C). A canCCV of AP1/ $11 \mathrm{~B}$ ko mice has wt levels of ITSN1 (Fig. 3). A stCCV has only 
$30 \%$ of a wt, stable CCV ITSN1 (Fig. 3). The constitutively active ITSN1 is the only synaptojanin 1 binding CCV protein, whose level is dramatically reduced in stCCV and thus ITSN1 appears to regulate synaptojanin $1 \mathrm{CCV}$ levels.

Preferential synaptojanin1 binding by ITSN1. In order to verify preferential ITSN1-synaptojanin1 binding within a CCV, we solubilised the CCV coats, immunoprecipitated ITSN1 and compared the amounts of co-isolated endophilin and synaptojanin1 between wt and ko synapse CCV. CCV have to be purified at $\mathrm{pH}$ 6.4 to stabilise the coat. Shifting the $\mathrm{pH}$ to 7-8 induces disassembly and solubilisation of the proteins. Vesicles are removed by centrifugation. The $\mathrm{pH}$ of the protein solution was adjusted back to 6.4 to restore the condition, which stabilises coat protein interactions. The ITSN1 antibody binds the SH3A-SH3B linker domain, competing with AP2 for ITSN1 binding ${ }^{73}$. Isolated anti-ITSN1antibody-beads were washed three times at $\mathrm{pH}$ 6.4. Proteins were eluted by adding threefold concentrated, dye-containing, non-reducing SDS-PAGE loading buffer. The amounts of proteins eluted from ko CCV ITSN1-beads were compared to those from wt CCV, also here defined as $100 \%$. Dynamin was not isolated (Fig. 4), indicating that ITSN1 and the co-isolated proteins are not part of a large protein network.

The wt and ko mouse canCCV have the same amount of ITSN1 (Fig. 3) and the same amounts of ITSN1 were immunoisolated (Fig. 4). A stCCV contains 30\% of wt, stable CCV ITSN1 and thus we expected to isolate less ITSN1 from stCCV, but stCCV eluates contained even slightly more ITSN1 than wt, stable CCV. Thus, ITSN1 immunoisolation efficiencies are CCV specific, for yet unkown reasons. The anti-ITSN1 antibody-AP2 competition for ITSN1 binding certainly contributes to this result ${ }^{73}$. However, the amounts of co-isolated synaptojanin 1 are CCV specific and thus the protein ratios do demonstrate CCV specific protein:protein interaction preferences. A ko synapse canCCV contains $40 \%$ and a stCCV contains 55\% of wt canCCV and stable CCV endophilin levels, but relatively more endophilin was co-isolated from both ko synapse CCV classes than from wt synapse CCV. Importantly, there is no difference in isolated endophilin levels between the wt and ko canCCV and the wt, stable CCV and the stCCV. This indicates a higher percentage of ITSN1-endophilin complexes in ko synapse CCV. This could be linked to the twofold stimulation of both CME pathways. Also these data speak against an endophilin function in the regulation of synaptojanin 1 CCV levels.

A ko synapse canCCV appears to have more ITSN1-synaptojanin1 complexes than a wt synapse canCCV, although the ko canCCV contains 50\% less synaptojanin 1 than a wt canCCV. This supports a critical function of ITSN1 in regulating synaptojanin 1 recruitment. Immunoisolation values indicate that stCCV have slightly fewer ITSN1-synaptojanin 1 complexes than wt, stable CCV (Fig. 4). However, a stCCV contains only $10 \%$ of synaptojanin1 of a wt, stable CCV. Therefore, much less synaptojanin1 would be isolated, if the ITSN1-synaptojanin 1 binding preference in stCCV would be identical with the preference in wt, stable CCV. Therefore, this experiment emphasises the regulation of synaptojanin1 levels by ITSN1 in CCV.

AP2 co-adaptors in ITSN1 CCV incorporation. Why do stCCV have so much less ITSN1? ITSN1 is bound by proteins initiating CCV formation: AP2, Eps15, Eps15L1, epsin and Sgip1 (Figs. 1B, 5A). Tripartite Sgip1-AP2-Eps15 and -Eps15L1 complexes support initiation of AP2 CCV budding, because Sgip1 binds low curvature membranes with highest affinity ${ }^{75-79}$. Epsin interacts with AP2 and ITSN1 ${ }^{80-85}$. Sgip1 binds, like synaptojanin1, to the ITSN1 SH3A domain. AP2 binds the ITSN1 SH3A-SH3B linker domain. All these proteins may compete for ITSN1 binding ${ }^{73}$. Eps15 and Eps15L1 bind the ITSN1 coiled-coil domain. Epsin and stonin2 bind the ITSN1 EH-domains ${ }^{74}$. Thus, protein:ITSN1 ratios of one of these proteins should determine the amount of ITSN1 in a CCV and thus we compared their levels between wt and ko CCV.

ITSN1 EH-domain binding proteins do not regulate ITSN1 CCV levels. Stonin2 CCV levels were compared previously ${ }^{1,2}$. It is present at wt levels in stCCV and therefore is not limiting for ITSN1 recruitment.

Epsin binds PI-4,5- $\mathrm{P}_{2}$ and ITSN1 (Fig. 5), AP2 and clathrin ${ }^{80,81,83,86,87}$. Epsin binds ubiquitinated cargo via its UIM domain (ubiquitin-interacting motif) ${ }^{85,88}$. Its ENTH domain dips in the cytoplasmic leaflet of the membrane, lowering the energy required for membrane bending ${ }^{89}$. CME is active in epsin ko MEF cells ${ }^{90}$. Thus, its prime function is to capture specific cargos ${ }^{91}$. We reasoned that its CCV levels might be even increased, because AP180, whose ANTH domain functions like the ENTH domain ${ }^{89}$, is reduced in ko canCCV to $60 \%$, and in

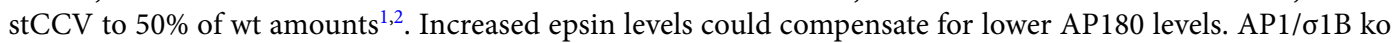
synapses have slightly more epsin (115\%) compared to wt synapses and a canCCV from ko mice has $30 \%$ of epsin compared to wt canCCV ${ }^{2}$. A stCCV has $65 \%$ of the epsin level of a wt, stable CCV (Fig. 5). Changes in epsin and in ITSN1 CCV levels go in opposite directions and thus epsin does not regulate ITSN1 recruitment. These data also demonstrate that AP2 CCV pathways are not enhanced to endocytose more epsin cargo proteins.

Sgip1 CCV levels are increased. Sgip1, a brain-specific homolog of the ubiquitous FcHO1/2 BARdomain protein, binds low curvature membranes with highest affinity, influencing CCV budding kinetics ${ }^{92,93}$.

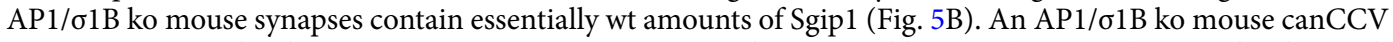
contains wt Sgip1 levels. stCCV contain even more Sgip1, than wt, stable CCV (Fig. 5B,C). Sgip1 levels match the AP2 CCV levels, indicating identical budding kinetics for both CCV classes in ko synapses. Thus, Sgip 1 does not determine CCV levels of ITSN1. Thus Eps15 and Eps15L1 might regulate ITSN1 recruitment and the CCV life cycle (Fig. 1).

Eps15, not Eps15L1, CCV levels regulate ITSN1 recruitment. Due to the formation of Sgip1-AP2Eps15 and -Eps15L1 complexes, Eps15 and/or Eps15L1 levels might be increased like the Sgip1 and AP2 levels. Eps15 and Eps15L1 bind the ITSN1 coiled-coil domain (Fig. 6) ${ }^{35}$. Eps15 ko mice are generally healthy and no 
A

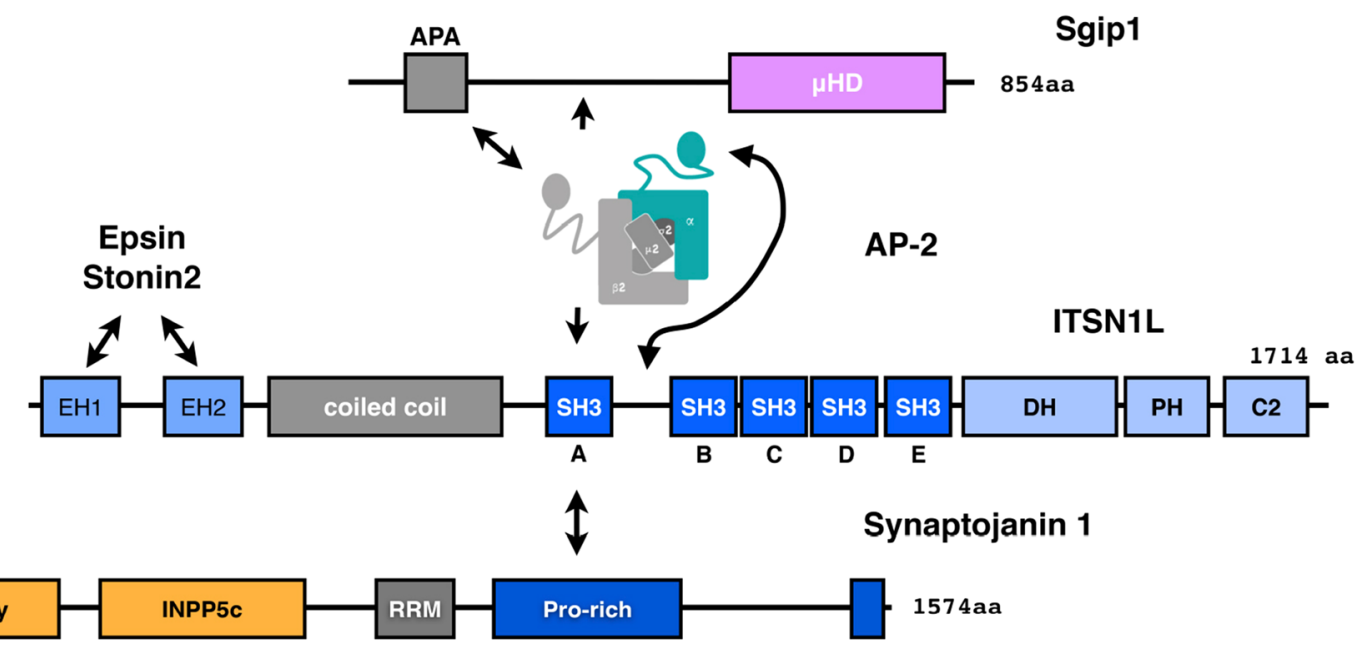

B
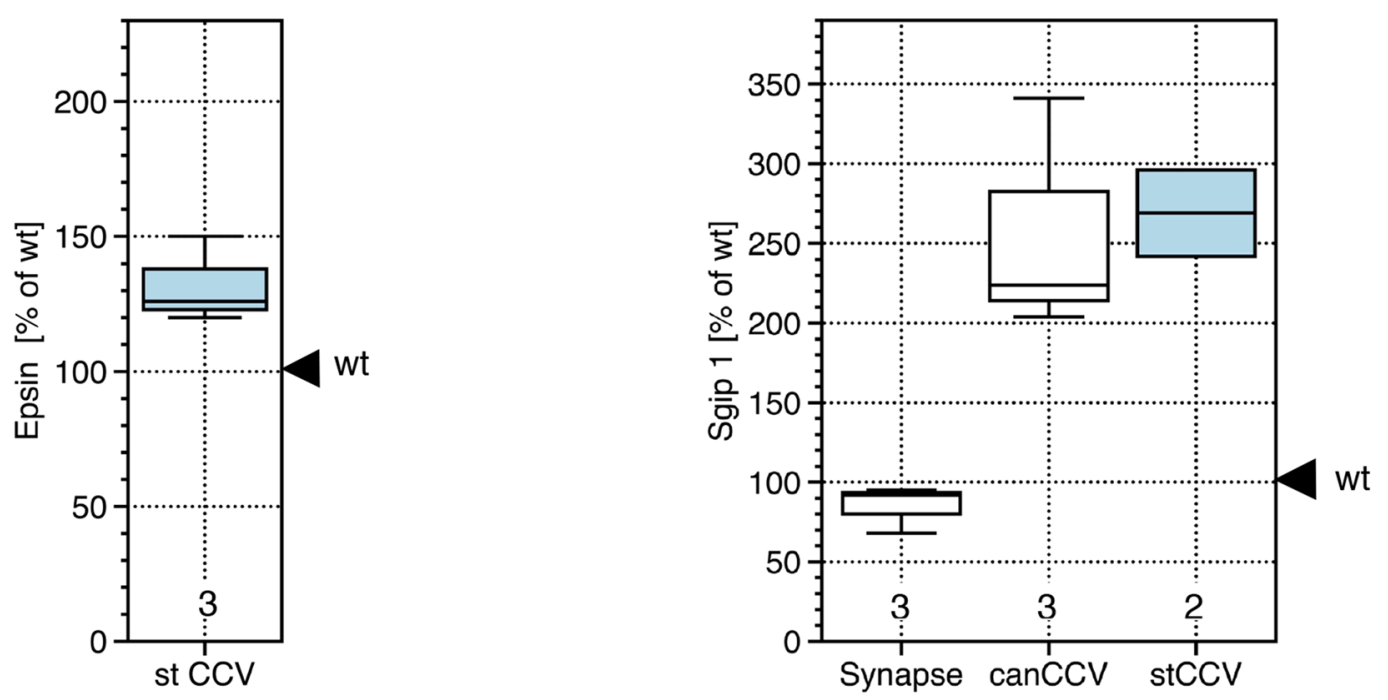

C

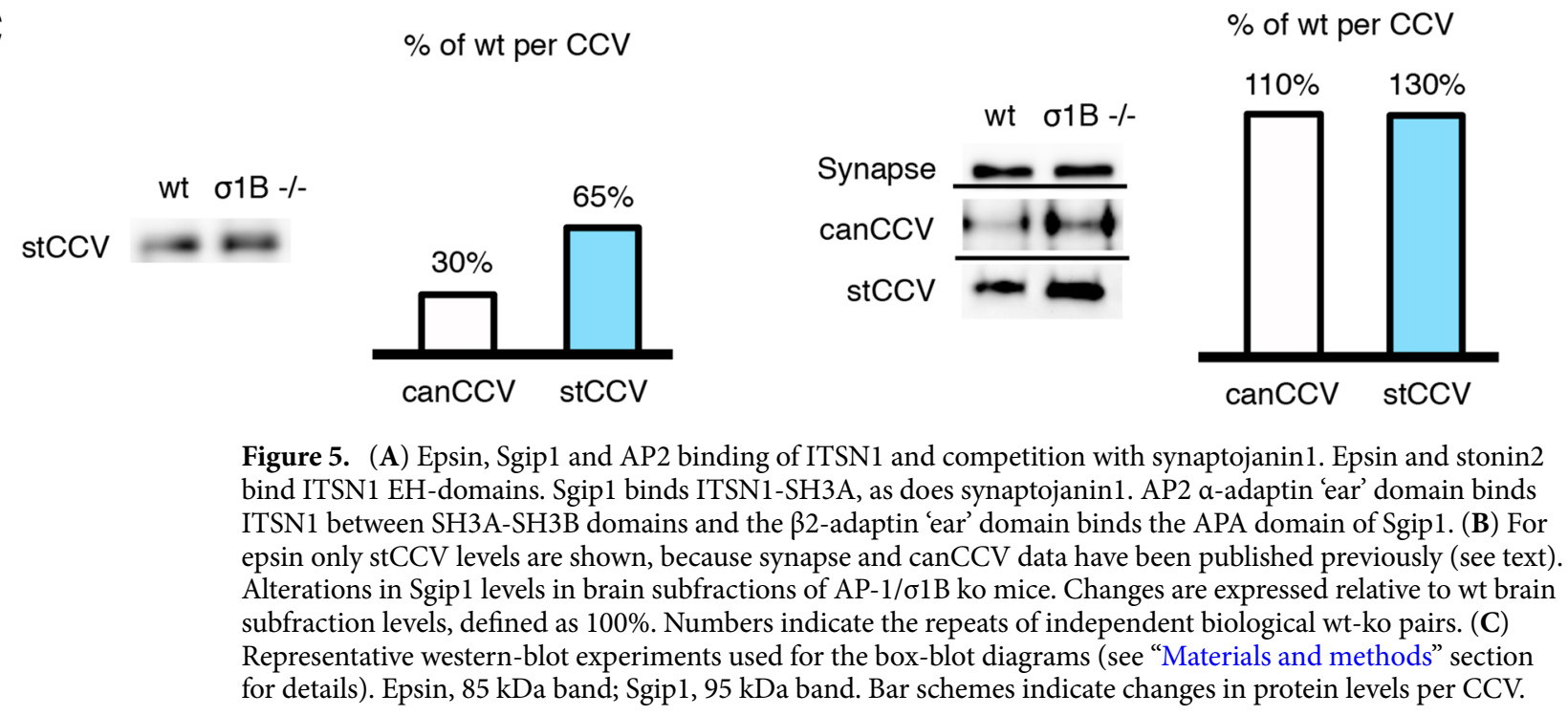

strong neurological phenotype has been described, although it is required for SV recycling ${ }^{94-96}$. Eps15L1 ko mice 
A

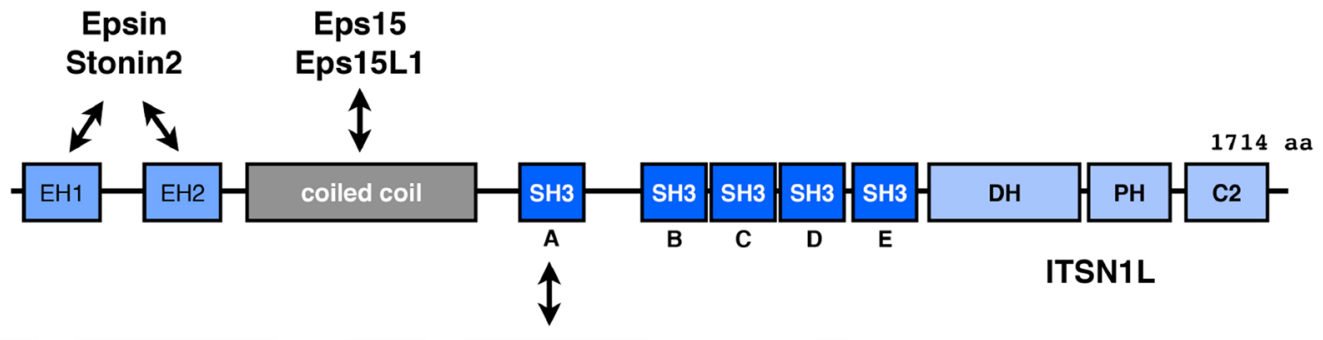

SAC-I homology

INPP5c

B

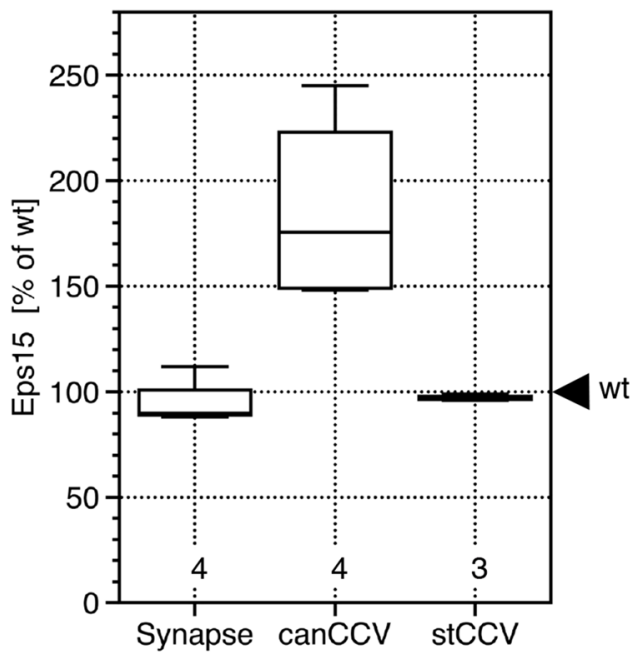

$\%$ of wt per CCV

C

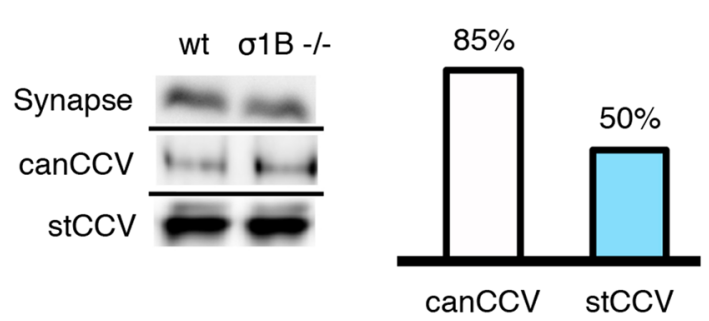

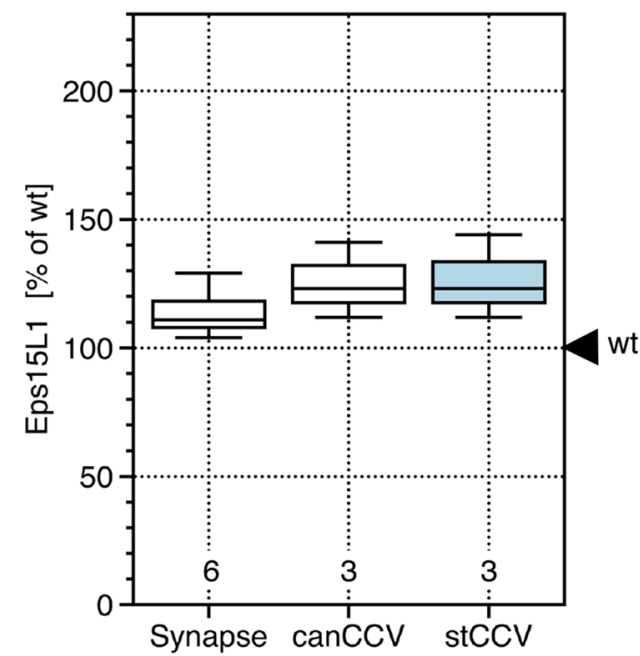

$\%$ of wt per CCV

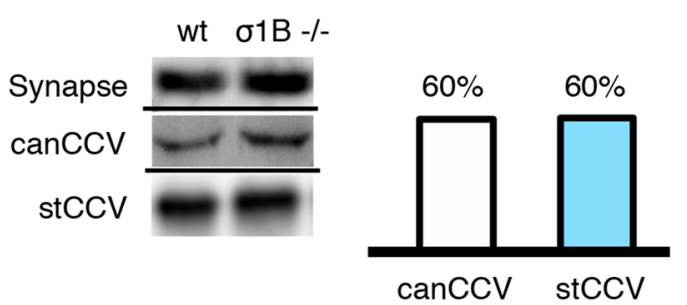

Figure 6. (A) ITSN1 domains bound by Eps15 and Eps15L1. (B) Alterations in Eps15 and Eps15L1 levels in

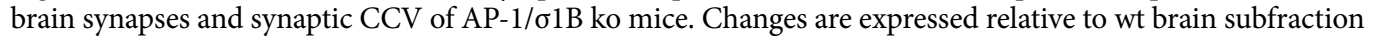
levels, defined as $100 \%$. Numbers indicate the repeats of independent biological wt-ko pairs. (C) Representative western-blot experiments used for the box-blot diagram (see "Materials and methods" section for details). Eps15, $140 \mathrm{kDa}$ band, Eps15L1 (Eps15R), $74 \mathrm{kDa}$ band. Bar schemes indicate changes in protein levels per CCV compared to wt set to $100 \%$.

die within two days after birth, showing severe neurological deficits. Thus, Eps15L1 appears to be able to substitute Eps15, but not vice versa ${ }^{97}$. Therefore, we expected CCV specific alterations in Eps15L1 rather than Eps15.

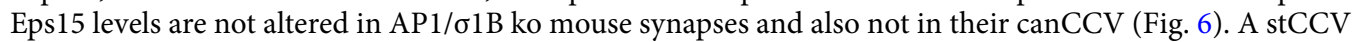
contains only $50 \%$ of the wt, stable CCV Eps 15 level. Eps15L1 levels are slightly increased in ko mice synapses (Fig. 6). Both CCV classes of ko mouse synapses contain 65\% of wt Eps15L1 levels. Although reduced, Eps15L1 incorporation into the CCV classes is not differentially regulated. Despite their high sequence homology, only Eps 15 recruitment into the CCV classes is differently regulated. Both are modified by phosphorylation, ubiquitination and acetylation and differential modifications might be responsible for this isoform specific regulation ${ }^{98,99}$. Of all ITSN1 binding CCV proteins, only Eps15 levels are specifically reduced in stCCV and thus Eps15 levels areis limiting for ITSN1 recruitment in a CCV (Fig. 7). 

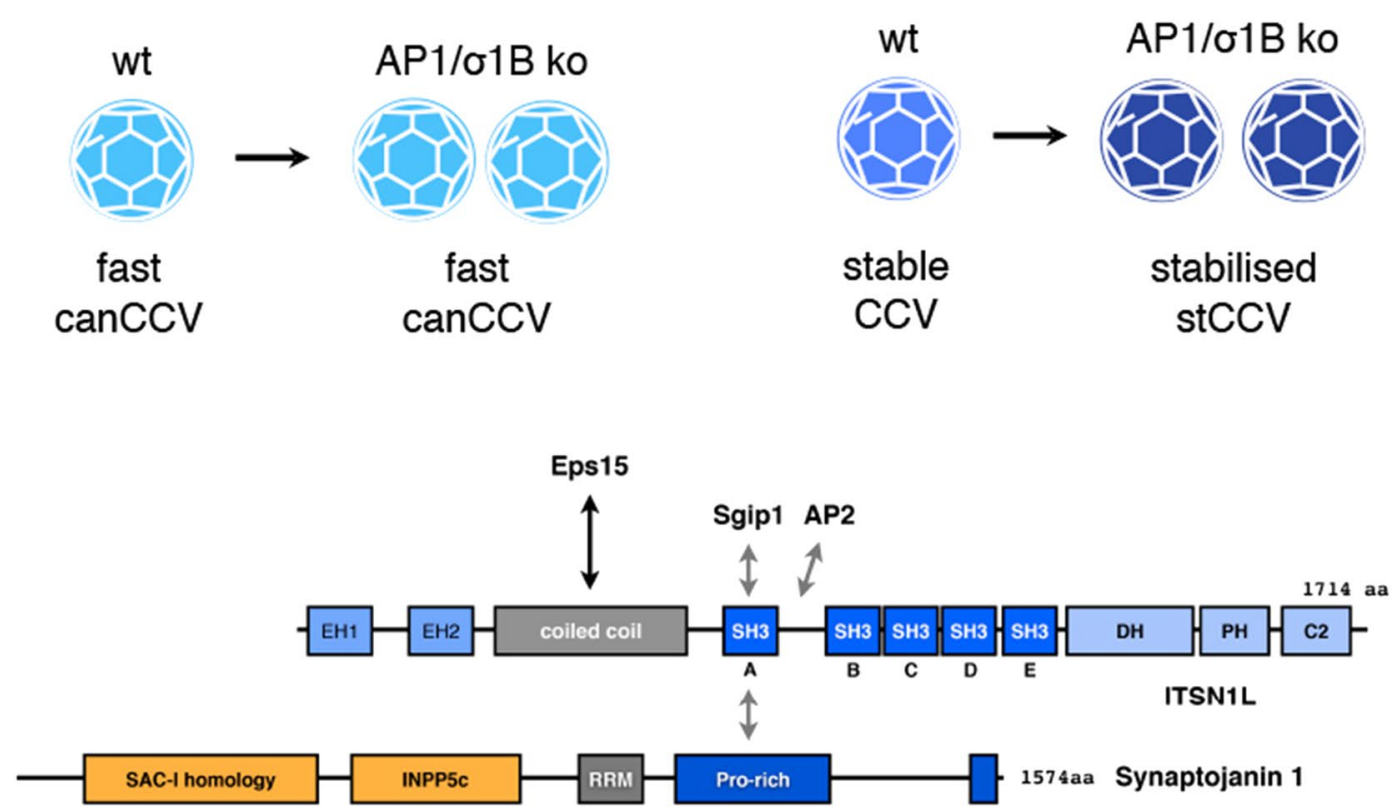

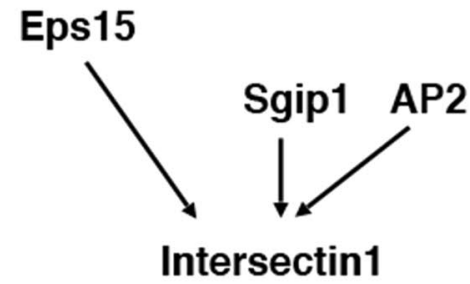

Synaptojanin 1

$\%$ of wt per canCCV

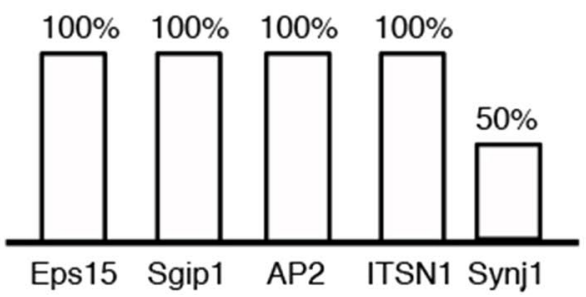

Eps15

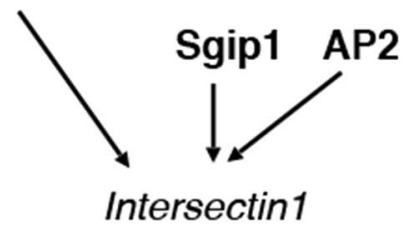

\section{Synaptojanin1}

$\%$ of wt per stCCV

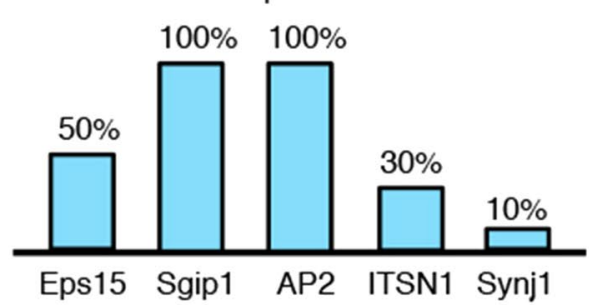

Figure 7. Model of protein-protein interactions determining the amount of synaptojanin1 stably incorporated into canCCV (left) and stCCV (right). Eps15 determines ITSN1 levels and the competition of Sgip1/AP2 and synaptojanin 1 for ITSN1 determines synaptojanin 1 levels. Interaction scheme: proteins in bold letters are present at $100 \%$ of wt levels, proteins in regular letters are reduced to $50 \%$ of wt levels, proteins in italics are reduced below $50 \%$ of wt levels. Please refer to the "Results" and "Discussion" sections for more details.

\section{Discussion}

We describe the analysis of synaptojanin 1 recruitment in the AP2 CCV coat, a mechanism which regulates the AP2 CCV life cycle. We have a unique experimental approach. We are using a mouse ko model, in which the AP2 CCV life cycle is modified as a secondary, indirect phenotype of the ko of the tissue-specific subunit $\sigma 1 \mathrm{~B}$ of the AP1 complex. The AP1/ $1 \mathrm{~B}$ ko causes alterations in synaptic protein sorting, which stimulate AP2 CCV mediated endocytosis and in addition the formation of a population of longer lived AP2 CCV. 
AP1/ $1 \mathrm{~B}$ deficiency causes severe learning and memory deficits in mice and severe intellectual disability in humans ${ }^{8}$. Synapses of ko mice have impaired SV recycling, reduced SV numbers and large early endosomes, whose maturation into late, MVB endosomes is stimulated ${ }^{1,2,7,8}$. The ko synapses have a twofold increase in the number of synaptic AP2 $\mathrm{CCV}^{2,100}$. This increase is synapse specific and therefore a mechanism of synaptic plasticity. Synapses contain two classes of AP2 CCV, canonical, canCCV $(\sim 85 \%)$, a second class $(\sim 15 \%)$ with a more stable coat. Both classes are increased twofold in the ko mice. A second induced mechanism of AP2 CCV plasticity is the enhanced stabilisation of the class of stable CCV in ko synapses, named stCCV. stCCV will have a longer half life and thus these endocytic vesicles are unable to fuse with an acceptor membrane shortly after their formation. They have a specific role in AZ plasticity ${ }^{1,2,7,8}$. These alterations in the AP2 CCV pathways are expected to suppress the deficits in synaptic signalling and brain functions caused by impaired AP1-dependent protein sorting.

The alterations in the AP2 CCV life cycle are physiological modifications of AP2 CCV composition. This enables us to study the mechanisms determining the composition of a mature AP2 CCV, without mutating CCV coat proteins. Overexpression or ko of CCV proteins allow only to study their earliest functions in the pathway, because coat proteins interact with several coat proteins during CCV formation and thus, multiple interactions are disturbed.

AP2 CCV pathway specific regulation of synaptojanin1 recruitment in CCV is one of the three described molecular mechanisms responsible for the formation of the stCCV: reduced recruitment of the uncoating ATPase Hsc70, hyperactivation of AP2 via increased amounts of AAK1 kinase and $\mu 2$ phosphorylation and thirdly, reduced recruitment of the PI-4,5- $\mathrm{P}_{2}$ phosphatase synaptojanin $1^{1}$. Synaptojanin 1 dysfunctions induce several, severe neurological diseases, including Alzheimer's and Parkinson's diseases and Down syndrome ${ }^{14,25,26,101,102}$. This demonstrates the importance of the tight regulation of synaptojanin 1 activities for brain function. Synaptojanin 1 catalytic activity is stimulated by high membrane curvature ${ }^{20}$. Therefore, mechanisms regulating its recruitment into CCV are most important.

Synaptojanin 1 interacts with 8 ubiquitously expressed CCV proteins, suggesting recruitment by several protein-protein interactions. Many of these proteins bind to each other forming a complex network (Figs. 1, 6). Of all the synaptojanin1 binding CCV proteins, endophilin has been considered to be most important for synaptojanin 1 recruitment and CCV uncoating, because it binds shortly before membrane scission together with synaptojanin $1^{13,43,58}$. It also appears reasonable, that an uncoating enzyme is recruited just before CCV formation is completed. However, endophilin CCV levels are not differentially altered between the CCV classes as are the synaptojanin 1 levels (Fig. 1) ${ }^{1}$. Our data also speak against AP2 CCV class specific regulation of endophilin protein interactions by LRRK2 kinase, whose mutations are very often the cause for Parkinson's disease ${ }^{52-54,103,104}$. However, our data do show that other endophilin CCV protein interactions do contribute to CCV life cycle regulation, because its binding to ITNS1 is enhanced in both ko CCV classes compared to wt CCV. This increased endophilin-ITSN1 interaction could be linked to the activation of both AP2 CCV pathways.

Pacsin 1 is a synaptojanin 1 binder, which connects CCV with the actin cytoskeleton ${ }^{64,67,105}$. Both canCCV contain the same amount of pacsin 1 , but stCCV contain only $30 \%$ of a wt, stable CCV However, this smaller pacsin 1 pool has a higher phosphorylation and thus activation ratio, than pacsin 1 of wt, stable CCV. The compensation of lower pacisn1 levels by an increased activation level is not in line with an impaired function and reduced synaptojanin 1 recruitment into stCCV. We are not able to completely exclude a pacsin 1 contribution to the regulation of synaptojanin1 recruitment. Unfortunately, the anti-pacsin1 antibodies do not allow efficient pacsin 1 immunoprecipitation and the quantification of co-isolated proteins by semi-quantitative western-blot experiments. The same experiment we did to verify the function of ITSN1 (Fig. 4). The pacsin1 data demonstrate remarkable complex regulatory mechanisms controlling CCV maturation. This quality control, sensor kinase has to be identified. Currently, we are analysing the additional two mechanisms responsible for stCCV formation, reduced Hsc70 binding and increased AAK1 kinase binding. We hope to identify also this pacsin1 kinase in the course of these studies. Pacsin 1 recruitment into stCCV is probably limited by an enriched coat protein, possibly the large scaffolding protein Git1.

The alterations in the CCV levels of the constitutively active synaptojanin 1 binder ITSN1 do match those of synaptojanin1. We verified that ITSN1 is indeed the major CCV protein binding synaptojanin 1 by immunoisolation of ITSN1 and determining the amounts of co isolated synaptojanin1. Importantly, the coat protein solutions used were from stripped CCV. Therefore, CCV proteins were present at the protein-protein ratios of a mature $\mathrm{CCV}$ and in their, possibly, post-translationally modified forms (see pacsin 1 data). ITSN1 binds synaptojanin 1 and endophilin independend of each other and it has been proposed, that ITSN1 coordinates the binding of both with each other and to the membrane ${ }^{58}$. Our data show that ITSN1 binds synaptojanin1 to regulate its CCV level and thus it takes part in the regulation of the CCV life cycle. The amount of synaptojanin 1 recruited by ITSN1 can be lowered by ITSN1 interaction with AP2/Sgip1. Sgip1 binds the ITSN1 SH3A domain, as does synaptojanin 1, and AP2 the SH3A-SH3B linker sequence. It has been proposed that they and synaptojanin 1 compete for ITSN1 binding (Figs. 5, 6) ${ }^{58}$. Sgip1 and AP2 are present in excess over ITSN1 in stCCV and thus are most likely responsible for the further reduction in synaptojanin 1 in stCCV. Synaptojanin 1 binding by the other CCV coat proteins is compared to ITSN1, either of to low affinity to determine the amount of synaptojanin 1 incorporated into CCV or the respective binding sites are sterically blocked or these proteins are modified and synaptojanin 1 binding is inhibited. There interactions with synaptojanin 1 could still have functions during CCV budding.

The next question to answer was, why do stCCV have so much less ITSN1 than wt, stable CCV? Of all possible ITSN1 recruiting CCV proteins, only the amount of Eps15 is differentially regulated between canCCV and stCCV. Eps15 and Eps15L1 bind the coiled-coil domain of ITSN1, located between EH and SH3 domains. This prevents ITSN1 homodimerisation and its inactivation ${ }^{74,106}$. It is remarkable that Eps15L1 is not involved in ISTN1 recruitment. This is surprising, because Eps15L1 ko mice show severe neurological defects, whereas Eps15 ko mice have comparably mild phenotypes. It seems that Eps15L1 is able to replace Eps15 in virtually any 
pathway ${ }^{97}$. Positions of functional domains are almost identical in Eps15 and Eps15L1 and thus there numerous phosphorylation and ubiquitination modifications should regulate isoform specific functions. The 50 amino acid long insertion in the Eps15L1 sequence is most frequently modified (summarised on PhosphoSitePlus). Analysis of coat proteome phosphorylation and dephosphorylation levels under different stimulation conditions of SV recycling, demonstrated functions in CCV and SV life cycle regulation ${ }^{107}$. Modifications might play a role in the regulation of CCV formation, but also in the regulation of the binding of specific cargo proteins ${ }^{98,99}$. Eps15 is also a cargo co-adapter CCV protein and therefore Eps15 specific cargo might determine the amount of Eps 15 recruited into CCV and Eps15 ability to recruit ITSN1. Alternatively, cargo and other coat proteins might interfere with Eps15 recruitment into CCV and/or its ability to bind ITSN1. For example, the stonin2 mediated recruitment of the large scaffolding protein Git1 into the stCCV endocytic pathway could alter coat protein-protein interactions ${ }^{1,3}$. This has to be tested in the future.

Collectively, our data lead to the model (Fig. 7), that Eps15 controls the amount of ITSN1 stably incorporated into CCV and that ITSN1 determines the amount of synaptojanin1 stably incorporated into AP2 CCV. Finally, the Sgip1/AP2:ITSN1 ratios determine how much synaptotagmin1 can stably bind to the ITSN1 incorporated in a CCV.

We discovered the variability of AP2 CCV life cycles as a mechanism of synaptic plasticity. The proteins involved are ubiquitously expressed and thus comparable mechanisms may play important roles in other tissues as well. However, the pool sizes of stabilised and longer lived CCV may be even smaller and more difficult to identify due to lower pathway activities. Synaptic plasticity is related to mechanisms in cell plasticity during development, which mediate cell-cell contact and tissue formation. Therefore, differential AP2 CCV life times may also be important during development and in tumors. The model of AP CCV coat protein interactions deduced from the presented data describes how AP2 co-adaptor proteins and thus AP2 CCV cargo-proteins determine the life cycle of their CCV.

\section{Materials and methods}

Isolation of synaptic canCCV and stCCV. The AP1/ $\sigma 1 \mathrm{~B}$ ko mouse has been described ${ }^{8,100,108}$ and also the isolation of synaptosomes and synaptic CCV has been described, which followed established protocols ${ }^{1,2}$. Animals are kept at the central animal facility of the University Medical Center, University Göttingen according to international guidelines. Animals were killed with $\mathrm{CO}_{2}$ and cervical dislocation in accordance with the appropriate guidelines. Animal housing and the protocol for killing the animals were approved by the, Niedersächsisches Landesamt für Verbraucherschutz und Lebensmittelsicherheit' (LAVES). Isolations from wt and AP1/ $\sigma 1 \mathrm{~B}-/$ - cortices were always performed in parallel and only data from wt and ko extracts prepared in parallel were compared with each other. Brains were isolated from 4 to 6 month old animals in late afternoon, snap frozen in liquid nitrogen and stored at $-80^{\circ} \mathrm{C}$. Isolation of stable CCV from wt and of stCCV from ko mice required 3 brains per genotype: sucrose density gradient fractions containing the purified synaptic CCV were pooled, and incubated with protein G Sepharose bead slurry (Protein G Sepharose 4 Fast Flow GE Healthcare) at $4{ }^{\circ} \mathrm{C}$ for $1 \mathrm{~h}$. Beads were pelleted at $2000 \mathrm{rpm}$ and the supernatant was incubated with $5 \mu \mathrm{g}$ of anti-Hsc70 mouse monoclonal antibody (Synaptic Systems, Göttingen, Ger) over night at $4{ }^{\circ} \mathrm{C}$. Protein G Sepharose beads were added at $4{ }^{\circ} \mathrm{C}$ for $4 \mathrm{~h}$. The harvested beads were washed twice with CCV buffer and resuspended in $40 \mu \mathrm{L} 3 \times$ SDS-PAGE loading buffer. The protein content of the beads and the wash fractions elution 1 and elution 2 were analysed by semi-quantitative western-blot analyses.

CCV coat protein quantification. Brain extracts were prepared from wt and ko mice in parallel and comparisons of protein content were only made between extracts prepared in parallel, which were separated next to each other on the same SDS-PAGE and transferred onto a nitrocellulose membrane (GE Healthcare Protran, $0.45 \mu \mathrm{m})$. All wt/ko data pairs are from independent biological samples. Comparing the data from different animals and independent preparations requires a normalisation and thus wt values were defined as $100 \%$. The protein load was varied between 10 and $80 \mu \mathrm{g}$ per lane to determine the linear protein/chemiluminescence signal ratio. Several proteins of different molecular masses were detected on one western-blot nitrocellulose membrane. This served as internal control for protein isolation and detection by the ECL luminescence detection kits PICO, NANO, FEMTO (Pierce-ThermoScientific, Karlsruhe, Ger), recorded with a Fuji LAS 1000 (Fujifilm Corp., Düsseldorf, Ger) camera system. Protein determination: Bradford-assay (BioRad, Munich, Ger) (Supplementary Material).

Coat protein: protein interactions. canCCV, wt stable CCV and stCCV were purified as described above. CCV coat disassembly of canCCV and of anti-Hsc70 antibody beads bound wt stable CCV and stCCV was induced by a pH shift from 6.4 to 8.0 in CCV buffer and incubation over $15 \mathrm{~min}$ at RT. Vesicles, beads and solubilised proteins were separated by centrifugation at $100.000 \times \mathrm{g}$ for $15 \mathrm{~min}$ at $4{ }^{\circ} \mathrm{C}$ (Beckman table-top ultracentrifuge). Supernatants containing solubilised CCV proteins were transferred into new tubes and the $\mathrm{pH}$ was adjusted back to $\mathrm{pH} 6.4$ to reestablish the conditions stabilising CCV protein:protein interactions. $5 \mu \mathrm{g}$ of the ITSN1 antibody was added to $1800 \mu \mathrm{L}$ supernatant and incubated overnight at $4{ }^{\circ} \mathrm{C}$. Protein-antibody complexes were isolated with Protein G sepharose-beads (GE Healthcare Life Sciences). Protein loaded beads were washed three times with $\mathrm{pH}$ 6.4 CCV buffer. Proteins were eluted by adding non-reducing, dye containing, $3 \times$ SDS-PAGE protein loading buffer. Elution fractions from $3 \mathrm{wt}$ and $3 \mathrm{ko}$ CCV-coIP isolations were pooled and loaded next to each other on SDS-PAGE gels and semi-quantitative western-blot analyses were performed as described above.

Statistics. All experiments were performed according to ARRIVE guidelines. The experiments were repeated at least three times with independent biological samples as described above. Wild-type and ko brain extracts were 
handled in parallel and values from wild-type samples were defined as $100 \%$ and the values of the ko samples processed in parallel are expressed relative to these $100 \%$. Only data sets in which all ko values are either above or below the respective wild-type values are considered to be significant. Statistics of the quantifications are presented as bar-plot diagrams, generated with DataGraph (Visual Data Tools, USA).

Antibodies. Antibodies: anti- $\alpha$-adaptin (AP2) (1:1000), anti-Eps15 (1:1000) and anti-ITSN1 (1:1000) were from BD Biosciences; anti-dynamin (1:1000), anti-Hsc70 (1:1000), anti-synaptojanin1 (1:300), its splice-variant (1:500), anti-endophilin A1 (1:1000) and anti-pacsin1 (1:1000) were from Synaptic Systems; anti-pacsin1 Ser346-Pi (1:1000) Merck Millipore; anti-LRRK2 (1:1000) Novus Biologicals, anti-ArhGEF7 (1:1000) GeneTex; anti-LRRK2 Ser935-Pi (1:1000) and anti-epsin1 (1:500) were from Abcam; anti-Sgip1 (1:500) Acris; antiEps15L1 (Eps15R) (1:500) Biorbyt; HRP-conjugated antibodies (1:10000): Dianova (Hamburg, Ger), anti-mouse (product \# 111-035-144), anti-rabbit (product \# 115-035-062); anti-goat (1:5000) (product \# 305-035-045).

Received: 7 August 2020; Accepted: 30 March 2021

Published online: 13 April 2021

\section{References}

1. Candiello, E., Mishra, R., Schmidt, B., Jahn, O. \& Schu, P. Differential regulation of synaptic AP-2/clathrin vesicle uncoating in synaptic plasticity. Sci. Rep. 7, 15781 (2017).

2. Kratzke, M., Candiello, E., Schmidt, B., Jahn, O. \& Schu, P. AP-1/б1B-dependent SV protein recycling is regulated in early endosomes and is coupled to AP-2 endocytosis. Mol. Neurobiol. 52, 142-161 (2015).

3. Podufall, J. et al. A presynaptic role for the cytomatrix protein GIT in synaptic vesicle recycling. Cell Rep. 7, 1417-1425 (2014).

4. Jung, N. et al. Molecular basis of synaptic vesicle cargo recognition by the endocytic sorting adaptor stonin 2. J. Cell Biol. 179, 1497-1510 (2007).

5. Diril, M. K., Wienisch, M., Jung, N., Klingauf, J. \& Haucke, V. Stonin 2 is an AP-2-dependent endocytic sorting adaptor for synaptotagmin internalization and recycling. Dev. Cell 10, 233-244 (2006).

6. Walther, K., Diril, M. K., Jung, N. \& Haucke, V. Functional dissection of the interactions of stonin 2 with the adaptor complex AP-2 and synaptotagmin. Proc. Natl. Acad. Sci. U.S.A. 101, 964-969 (2004).

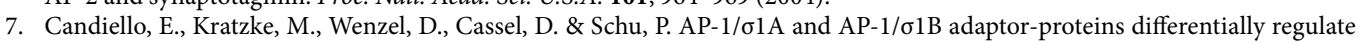
neuronal early endosome maturation via the Rab5/Vps34-pathway. Sci. Rep. 6, 29950 (2016).

8. Glyvuk, N. et al. AP-1/o1B-adaptin mediates endosomal synaptic vesicle recycling, learning and memory. EMBO J. 29, 1318-1330 (2010).

9. Henderson, D. M. \& Conner, S. D. A novel AAK1 splice variant functions at multiple steps of the endocytic pathway. Mol. Biol. Cell 18, 2698-2706 (2007).

10. Conner, S. D. \& Schmid, S. L. Identification of an adaptor-associated kinase, AAK1, as a regulator of clathrin-mediated endocytosis. J. Cell Biol. 156, 921-929 (2002).

11. Ricotta, D., Conner, S. D., Schmid, S. L., von Figura, K. \& Höning, S. Phosphorylation of the AP2 $\mu$ subunit by AAK1 mediates high affinity binding to membrane protein sorting signals. J. Cell Biol. 156, 791-795 (2002).

12. Jackson, L. P. et al. A large-scale conformational change couples membrane recruitment to cargo binding in the AP2 clathrin adaptor complex. Cell 141, 1220-1229 (2010).

13. Milosevic, I. et al. Recruitment of endophilin to clathrin-coated pit necks is required for efficient vesicle uncoating after fission. Neuron 72, 587-601 (2011).

14. Cao, M. et al. Parkinson sac domain mutation in synaptojanin 1 impairs clathrin uncoating at synapses and triggers dystrophic changes in dopaminergic axons. Neuron $\mathbf{9 3}, 882-896$ (2017).

15. Ramjaun, A. R. \& McPherson, P. S. Tissue-specific alternative splicing generates two synaptojanin isoforms with differential membrane binding properties. J. Biol. Chem. 271, 24856-24861 (1996).

16. Khvotchev, M. \& Sudhof, T. C. Developmentally regulated alternative splicing in a novel synaptojanin. J. Biol. Chem. 273, 2306-2311 (1998).

17. Harris, T. W., Hartwieg, E., Horvitz, H. R. \& Jorgensen, E. M. Mutations in synaptojanin disrupt synaptic vesicle recycling. J. Cell Biol. 150, 589-600 (2000).

18. Perera, R. M., Zoncu, R., Lucast, L., De Camilli, P. \& Toomre, D. Two synaptojanin 1 isoforms are recruited to clathrin-coated pits at different stages. Proc. Natl. Acad. Sci. U.S.A. 103, 19332-19337 (2006).

19. Mani, M. et al. The dual phosphatase activity of synaptojanin1 is required for both efficient synaptic vesicle endocytosis and reavailability at nerve terminals. Neuron 56, 1004-1018 (2007).

20. Chang-Ileto, B. et al. Synaptojanin 1-mediated PI(4,5)P2 hydrolysis is modulated by membrane curvature and facilitates membrane fission. Dev. Cell 20, 206-218 (2011).

21. Hardies, K. et al. Loss of SYNJ1 dual phosphatase activity leads to early onset refractory seizures and progressive neurological decline. Brain 139, 2420-2430 (2016).

22. Krebs, C. E. et al. The Sacl domain of SYNJ1 identified mutated in a family with early-onset progressive Parkinsonism with generalized seizures. Hum. Mutat. 34, 1200-1207 (2013).

23. Quadri, M. et al. Mutation in the SYNJ1 gene associated with autosomal recessive, early-onset Parkinsonism. Hum. Mutat. 34, 1208-1215 (2013).

24. Dyment, D. A. et al. Homozygous nonsense mutation in SYNJ1 associated with intractable epilepsy and tau pathology. Neurobiol. Aging 36(1222), e1221-1225 (2015).

25. Rauschendorf, M. A. et al. Novel compound heterozygous synaptojanin-1 mutation causes l-dopa-responsive dystonia-parkinsonism syndrome. Mov. Disord. 32, 478-480 (2017).

26. Ben Romdhan, S. et al. A novel SYNJ1 mutation in a tunisian family with juvenile Parkinson's disease associated with epilepsy. J. Mol. Neurosci. 66, 273-278 (2018).

27. Zhu, L. et al. Reduction of synaptojanin 1 accelerates Abeta clearance and attenuates cognitive deterioration in an Alzheimer mouse model. J. Biol. Chem. 288, 32050-32063 (2013).

28. McIntire, L. B. et al. Reduction of synaptojanin 1 ameliorates synaptic and behavioral impairments in a mouse model of Alzheimer's disease. J. Neurosci. 32, 15271-15276 (2012).

29. Martin, S. B. et al. Synaptophysin and synaptojanin-1 in Down syndrome are differentially affected by Alzheimer's disease. J. Alzheimers Dis. 42, 767-775 (2014). 
30. Arai, Y., Ijuin, T., Takenawa, T., Becker, L. E. \& Takashima, S. Excessive expression of synaptojanin in brains with Down syndrome. Brain Dev. 24, 67-72 (2002).

31. Voronov, S. V. et al. Synaptojanin 1-linked phosphoinositide dyshomeostasis and cognitive deficits in mouse models of Down's syndrome. Proc. Natl. Acad. Sci. U.S.A. 105, 9415-9420 (2008).

32. Vanhauwaert, R. et al. The SAC1 domain in synaptojanin is required for autophagosome maturation at presynaptic terminals. EMBO J. 36, 1392-1411 (2017).

33. George, A. A., Hayden, S., Stanton, G. R. \& Brockerhoff, S. E. Arf6 and the 5'phosphatase of Synaptojanin 1 regulate autophagy in cone photoreceptors. Inside Cell 1, 117-133 (2016).

34. Haffner, C., Di Paolo, G., Rosenthal, J. A. \& de Camilli, P. Direct interaction of the $170 \mathrm{kDa}$ isoform of synaptojanin 1 with clathrin and with the clathrin adaptor AP-2. Curr. Biol. 10, 471-474 (2000).

35. Haffner, C. et al. Synaptojanin 1: Localization on coated endocytic intermediates in nerve terminals and interaction of its 170 $\mathrm{kDa}$ isoform with Eps15. FEBS Lett. 419, 175-180 (1997).

36. Jha, A. et al. A novel AP-2 adaptor interaction motif initially identified in the long-splice isoform of synaptojanin 1, SJ170. J. Biol. Chem. 279, 2281-2290 (2004).

37. Micheva, K. D., Kay, B. K. \& McPherson, P. S. Synaptojanin forms two separate complexes in the nerve terminal. Interactions with endophilin and amphiphysin. J. Biol. Chem. 272, 27239-27245 (1997).

38. Cestra, G. et al. The SH3 domains of endophilin and amphiphysin bind to the proline-rich region of synaptojanin 1 at distinct sites that display an unconventional binding specificity. J. Biol. Chem. 274, 32001-32007 (1999).

39. Modregger, J., Ritter, B., Witter, B., Paulsson, M. \& Plomann, M. All three PACSIN isoforms bind to endocytic proteins and inhibit endocytosis. J. Cell Sci. 113, 4511-4521 (2000).

40. Zucconi, A., Dente, L., Santonico, E., Castagnoli, L. \& Cesareni, G. Selection of ligands by panning of domain libraries displayed on phage lambda reveals new potential partners of synaptojanin 1. J. Mol. Biol. 307, 1329-1339 (2001).

41. Verstreken, P. et al. Synaptojanin is recruited by endophilin to promote synaptic vesicle uncoating. Neuron 40, 733-748 (2003).

42. Schuske, K. R. et al. Endophilin is required for synaptic vesicle endocytosis by localizing synaptojanin. Neuron 40, 749-762 (2003).

43. Dong, Y., Gou, Y., Li, Y., Liu, Y. \& Bai, J. Synaptojanin cooperates in vivo with endophilin through an unexpected mechanism. Elife. https://doi.org/10.7554/eLife.05660 (2015).

44. Kim, W. T. et al. Delayed reentry of recycling vesicles into the fusion-competent synaptic vesicle pool in synaptojanin 1 knockout mice. Proc. Natl. Acad. Sci. U.S.A. 99, 17143-17148 (2002).

45. Luthi, A. et al. Synaptojanin 1 contributes to maintaining the stability of GABAergic transmission in primary cultures of cortical neurons. J. Neurosci. 21, 9101-9111 (2001).

46. Cremona, O. et al. Essential role of phosphoinositide metabolism in synaptic vesicle recycling. Cell 99, 179-188 (1999).

47. Cousin, M. A., Tan, T. C. \& Robinson, P. J. Protein phosphorylation is required for endocytosis in nerve terminals: Potential role for the dephosphins dynamin I and synaptojanin, but not AP180 or amphiphysin. J. Neurochem. 76, 105-116 (2001).

48. Lee, S. Y., Wenk, M. R., Kim, Y., Nairn, A. C. \& De Camilli, P. Regulation of synaptojanin 1 by cyclin-dependent kinase 5 at synapses. Proc. Natl. Acad. Sci. U.S.A. 101, 546-551 (2004).

49. Irie, F., Okuno, M., Pasquale, E. B. \& Yamaguchi, Y. EphrinB-EphB signalling regulates clathrin-mediated endocytosis through tyrosine phosphorylation of synaptojanin 1. Nat. Cell Biol. 7, 501-509 (2005).

50. Strochlic, T. I. et al. Identification of neuronal substrates implicates Pak5 in synaptic vesicle trafficking. Proc. Natl. Acad. Sci. U.S.A. 109, 4116-4121 (2012).

51. Chen, C. K. et al. Activity-dependent facilitation of Synaptojanin and synaptic vesicle recycling by the Minibrain kinase. Nat. Commun. 5, 4246 (2014).

52. Alessi, D. R. \& Sammler, E. LRRK2 kinase in Parkinson's disease. Science 360, 36-37 (2018).

53. Matta, S. et al. LRRK2 controls an EndoA phosphorylation cycle in synaptic endocytosis. Neuron 75, 1008-1021 (2012).

54. Piccoli, G. et al. LRRK2 controls synaptic vesicle storage and mobilization within the recycling pool. J. Neurosci. 31, 2225-2237 (2011).

55. Gallop, J. L. et al. Mechanism of endophilin N-BAR domain-mediated membrane curvature. EMBO J. 25, 2898-2910 (2006).

56. Zhang, X. M. et al. A proline-rich motif on VGLUT1 reduces synaptic vesicle super-pool and spontaneous release frequency. Elife. https://doi.org/10.7554/eLife.50401 (2019).

57. Hohendahl, A. et al. Structural inhibition of dynamin-mediated membrane fission by endophilin. Elife. https://doi.org/10.7554/ eLife.26856 (2017).

58. Pechstein, A. et al. Vesicle uncoating regulated by SH3-SH3 domain-mediated complex formation between endophilin and intersectin at synapses. EMBO Rep. 16, 232-239 (2015).

59. Tian, Q., Zhang, J. F., Fan, J., Song, Z. \& Chen, Y. Endophilin isoforms have distinct characteristics in interactions with N-type Ca2+ channels and dynamin I. Neurosci. Bull. 28, 483-492 (2012).

60. Sundborger, A. et al. An endophilin-dynamin complex promotes budding of clathrin-coated vesicles during synaptic vesicle recycling. J. Cell Sci. 124, 133-143 (2011).

61. Boulakirba, S. et al. Arf6 exchange factor EFA6 and endophilin directly interact at the plasma membrane to control clathrinmediated endocytosis. Proc. Natl. Acad. Sci. U.S.A. 111, 9473-9478 (2014).

62. Stevers, L. M. et al. Structural interface between LRRK2 and 14-3-3 protein. Biochem. J. 474, 1273-1287 (2017).

63. Li, X. et al. Phosphorylation-dependent 14-3-3 binding to LRRK2 is impaired by common mutations of familial Parkinson's disease. PLoS ONE 6, e17153 (2011)

64. Quan, A. \& Robinson, P. J. Syndapin-a membrane remodelling and endocytic F-BAR protein. FEBS J. 280, 5198-5212 (2013).

65. Schael, S. et al. Casein kinase 2 phosphorylation of protein kinase $C$ and casein kinase 2 substrate in neurons (PACSIN) 1 protein regulates neuronal spine formation. J. Biol. Chem. 288, 9303-9312 (2013).

66. Quan, A. et al. Phosphorylation of syndapin I F-BAR domain at two helix-capping motifs regulates membrane tubulation. Proc. Natl. Acad. Sci. U.S.A. 109, 3760-3765 (2012).

67. Halbach, A. et al. PACSIN 1 forms tetramers via its N-terminal F-BAR domain. FEBS J. 274, 773-782 (2007).

68. Schulze, W. X. \& Mann, M. A novel proteomic screen for peptide-protein interactions. J. Biol. Chem. 279, 10756-10764 (2004).

69. Huttlin, E. L. et al. A tissue-specific atlas of mouse protein phosphorylation and expression. Cell 143, 1174-1189 (2010).

70. Pan, P. Y. et al. Parkinson's disease-associated LRRK2 hyperactive kinase mutant disrupts synaptic vesicle trafficking in ventral midbrain neurons. J. Neurosci. 37, 11366-11376 (2017).

71. Gerth, F. et al. Exon inclusion modulates conformational plasticity and autoinhibition of the intersectin 1 SH3A domain. Structure 27, 977 (2019).

72. Hussain, N. K. et al. Splice variants of intersectin are components of the endocytic machinery in neurons and nonneuronal cells. J. Biol. Chem. 274, 15671-15677 (1999).

73. Pechstein, A. et al. Regulation of synaptic vesicle recycling by complex formation between intersectin 1 and the clathrin adaptor complex AP2. Proc. Natl. Acad. Sci. U.S.A. 107, 4206-4211 (2010).

74. Pechstein, A., Shupliakov, O. \& Haucke, V. Intersectin 1: A versatile actor in the synaptic vesicle cycle. Biochem. Soc. Trans. 38, $181-186(2010)$ 
75. Zhang, Y., Feng, Y., Xin, Y. \& Liu, X. SGIP1 dimerizes via intermolecular disulfide bond in muHD domain during cellular endocytosis. Biochem. Biophys. Res. Commun. 505, 99-105 (2018).

76. Shimada, A., Yamaguchi, A. \& Kohda, D. Structural basis for the recognition of two consecutive mutually interacting DPF motifs by the SGIP1 mu homology domain. Sci. Rep. 6, 19565 (2016).

77. Hollopeter, G. et al. The membrane-associated proteins FCHo and SGIP are allosteric activators of the AP2 clathrin adaptor complex. Elife. https://doi.org/10.7554/eLife.03648 (2014).

78. Li, H. D., Liu, W. X. \& Michalak, M. Enhanced clathrin-dependent endocytosis in the absence of calnexin. PLoS ONE 6, e21678 (2011).

79. Uezu, A. et al. SGIP1alpha is an endocytic protein that directly interacts with phospholipids and Eps15. J. Biol. Chem. 282, 26481-26489 (2007).

80. Drake, M. T., Downs, M. A. \& Traub, L. M. Epsin binds to clathrin by associating directly with the clathrin-terminal domain. Evidence for cooperative binding through two discrete sites. J. Biol. Chem. 275, 6479-6489 (2000).

81. Owen, D. J., Vallis, Y., Pearse, B. M., McMahon, H. T. \& Evans, P. R. The structure and function of the $\beta 2$-adaptin appendage domain. EMBO J. 19, 4216-4227 (2000).

82. Kalthoff, C., Alves, J., Urbanke, C., Knorr, R. \& Ungewickell, E. J. Unusual structural organization of the endocytic proteins AP180 and epsin 1. J. Biol. Chem. 277, 8209-8216 (2002).

83. Brett, T. J., Traub, L. M. \& Fremont, D. H. Accessory protein recruitment motifs in clathrin-mediated endocytosis. Structure 10, 797-809 (2002).

84. Edeling, M. A. et al. Molecular switches involving the AP-2 $\beta 2$ appendage regulate endocytic cargo selection and clathrin coat assembly. Dev. Cell 10, 329-342 (2006).

85. Hawryluk, M. J. et al. Epsin 1 is a polyubiquitin-selective clathrin-associated sorting protein. Traffic 7, 262-281 (2006).

86. Ford, M. G. et al. Curvature of clathrin-coated pits driven by epsin. Nature 419, 361-366 (2002).

87. Kyung, J. W., Bae, J. R., Kim, D. H., Song, W. K. \& Kim, S. H. Epsin1 modulates synaptic vesicle retrieval capacity at CNS synapses. Sci. Rep. 6, 31997 (2016).

88. Kazazic, M. et al. Epsin 1 is involved in recruitment of ubiquitinated EGF receptors into clathrin-coated pits. Traffic 10, 235-245 (2009).

89. Hom, R. A. et al. pH-dependent binding of the Epsin ENTH domain and the AP180 ANTH domain to PI(4,5)P2-containing bilayers. J. Mol. Biol. 373, 412-423 (2007).

90. Messa, M. et al. Epsin deficiency impairs endocytosis by stalling the actin-dependent invagination of endocytic clathrin-coated pits. Elife 3, e03311 (2014).

91. Chen, H. et al. Embryonic arrest at midgestation and disruption of Notch signaling produced by the absence of both epsin 1 and epsin 2 in mice. Proc. Natl. Acad. Sci. U.S.A. 106, 13838-13843 (2009).

92. Haucke, V. \& Kozlov, M. M. Membrane remodeling in clathrin-mediated endocytosis. J. Cell Sci. 131, 21681 (2018).

93. McMahon, H. T. \& Boucrot, E. Membrane curvature at a glance. J. Cell Sci. 128, 1065-1070 (2015).

94. Pozzi, B. et al. The endocytic adaptor Eps15 controls marginal zone B cell numbers. PLoS ONE 7, e50818 (2012).

95. Koh, T. W. et al. Eps15 and Dap160 control synaptic vesicle membrane retrieval and synapse development. J. Cell Biol. 178, 309-322 (2007).

96. Salcini, A. E. et al. The Eps15 C. elegans homologue EHS-1 is implicated in synaptic vesicle recycling. Nat. Cell Biol. 3, 755-760 (2001).

97. Milesi, C. et al. Redundant and nonredundant organismal functions of EPS15 and EPS15L1. Life Sci. Allian. 2, e201800273 (2019).

98. Gschweitl, M. et al. A SPOPL/Cullin-3 ubiquitin ligase complex regulates endocytic trafficking by targeting EPS15 at endosomes. Elife 5, e13841 (2016).

99. Traub, L. M. A nanobody-based molecular toolkit provides new mechanistic insight into clathrin-coat initiation. Elife. https:// doi.org/10.7554/eLife.41768 (2019).

100. Baltes, J. et al. $\sigma 1 \mathrm{~B}$ adaptin regulates adipogenesis by mediating the sorting of sortilin in adipose tissue. J. Cell Sci. 127, 3477-3487 (2014).

101. Al Zaabi, N., Al Menhali, N. \& Al-Jasmi, F. SYNJ1 gene associated with neonatal onset of neurodegenerative disorder and intractable seizure. Mol. Genet. Genomic Med. 6, 109-113 (2018).

102. Miranda, A. M. et al. Excess synaptojanin 1 contributes to place cell dysfunction and memory deficits in the aging hippocampus in three types of Alzheimer's disease. Cell Rep. 23, 2967-2975 (2018).

103. Nguyen, M. \& Krainc, D. LRRK2 phosphorylation of auxilin mediates synaptic defects in dopaminergic neurons from patients with Parkinson's disease. Proc. Natl. Acad. Sci. U.S.A. 115, 5576-5581 (2018).

104. Arranz, A. M. et al. LRRK2 functions in synaptic vesicle endocytosis through a kinase-dependent mechanism. J. Cell Sci. 128, 541-552 (2015).

105. Qualmann, B. \& Kelly, R. B. Syndapin isoforms participate in receptor-mediated endocytosis and actin organization. J. Cell Biol. 148, 1047-1062 (2000).

106. Gubar, O. et al. Intersectin: The crossroad between vesicle exocytosis and endocytosis. Front. Endocrinol. (Lausanne) 4, 109 (2013).

107. Kohansal-Nodehi, M., Chua, J. J., Urlaub, H., Jahn, R. \& Czernik, D. Analysis of protein phosphorylation in nerve terminal reveals extensive changes in active zone proteins upon exocytosis. Elife. https://doi.org/10.7554/eLife.14530 (2016).

108. Zizioli, D. et al. $\gamma 2$ and $\gamma 1$ AP-1 complexes: Different essential functions and regulatory mechanisms in clathrin-dependent protein sorting. Eur. J. Cell Biol. 96, 356-368 (2017).

\section{Acknowledgements}

This work was supported by Grants DFG Schu 802/3-2, /3-4 and /3-5 to PS and GGNB grants to R.M. and E.C.

\section{Author contributions}

R.M., G.F.S. and E.C. performed and analysed the experiments and P.S. conceived and analysed the experiments and wrote the manuscript.

\section{Funding}

Open Access funding enabled and organized by Projekt DEAL.

\section{Competing interests}

The authors declare no competing interests. 


\section{Additional information}

Supplementary Information The online version contains supplementary material available at https://doi.org/ 10.1038/s41598-021-87591-3.

Correspondence and requests for materials should be addressed to P.S.

Reprints and permissions information is available at www.nature.com/reprints.

Publisher's note Springer Nature remains neutral with regard to jurisdictional claims in published maps and institutional affiliations.

(c) (1) Open Access This article is licensed under a Creative Commons Attribution 4.0 International cc) License, which permits use, sharing, adaptation, distribution and reproduction in any medium or format, as long as you give appropriate credit to the original author(s) and the source, provide a link to the Creative Commons licence, and indicate if changes were made. The images or other third party material in this article are included in the article's Creative Commons licence, unless indicated otherwise in a credit line to the material. If material is not included in the article's Creative Commons licence and your intended use is not permitted by statutory regulation or exceeds the permitted use, you will need to obtain permission directly from the copyright holder. To view a copy of this licence, visit http://creativecommons.org/licenses/by/4.0/.

(C) The Author(s) 2021 